\title{
How students process equations in solving quantitative synthesis problems? Role of mathematical complexity in students' mathematical performance
}

\author{
Bashirah Ibrahim, ${ }^{1}$ Lin Ding, ${ }^{1, *}$ Andrew F. Heckler, ${ }^{2, \dagger}$ Daniel R. White, ${ }^{2}$ and Ryan Badeau ${ }^{2}$ \\ ${ }^{1}$ Department of Teaching and Learning, The Ohio State University, Columbus, Ohio 43210, USA \\ ${ }^{2}$ Department of Physics, The Ohio State University, Columbus, Ohio 43210, USA
}

(Received 10 March 2017; published 5 October 2017)

\begin{abstract}
We examine students' mathematical performance on quantitative "synthesis problems" with varying mathematical complexity. Synthesis problems are tasks comprising multiple concepts typically taught in different chapters. Mathematical performance refers to the formulation, combination, and simplification of equations. Generally speaking, formulation and combination of equations require conceptual reasoning; simplification of equations requires manipulation of equations as computational tools. Mathematical complexity is operationally defined by the number and the type of equations to be manipulated concurrently due to the number of unknowns in each equation. We use two types of synthesis problems, namely, sequential and simultaneous tasks. Sequential synthesis tasks require a chronological application of pertinent concepts, and simultaneous synthesis tasks require a concurrent application of the pertinent concepts. A total of 179 physics major students from a second year mechanics course participated in the study. Data were collected from written tasks and individual interviews. Results show that mathematical complexity negatively influences the students' mathematical performance on both types of synthesis problems. However, for the sequential synthesis tasks, it interferes only with the students' simplification of equations. For the simultaneous synthesis tasks, mathematical complexity additionally impedes the students' formulation and combination of equations. Several reasons may explain this difference, including the students' different approaches to the two types of synthesis problems, cognitive load, and the variation of mathematical complexity within each synthesis type.
\end{abstract}

DOI: 10.1103/PhysRevPhysEducRes.13.020120

\section{INTRODUCTION}

Quantitative physics problem solving usually involves the blending of concepts and mathematics. Most physics problems, including traditional end-of-chapter exercises in textbooks, are structured to comprise a conceptual aspect (the underlying physics concepts and their application) and a mathematical aspect (manipulation of equations and mathematical tools). Although mathematics is an essential component of physics, its manipulation often occurs mechanistically. Students view equations as computational tools and struggle to ascribe meaning to the mathematical symbols and tools as used in the physics domain [1-4].

A number of studies on problem solving have focused on how students use mathematics when tackling physics problems. These studies were implemented with students in lower and upper division calculus-based physics courses. Students'

\footnotetext{
Corresponding author. ding.65@osu.edu

Corresponding author. heckler.6@osu.edu

Published by the American Physical Society under the terms of the Creative Commons Attribution 4.0 International license. Further distribution of this work must maintain attribution to the author(s) and the published article's title, journal citation, and DOI.
}

reasoning when manipulating the mathematics of physics problems was elicited using the framework of epistemic games [5-7], resources [8,9], epistemological framing $[2,10,11]$, cognitive, or conceptual blending [12-14]. Their difficulties when using mathematical tools, such as integrals and partial derivatives, in the physics context were also identified [3,14-21]. Further, studies were conducted to explore the link between students' mathematical difficulties and their understanding of physics concepts [22,23]. Research has also demonstrated that using mathematical concepts, tools, and procedures in pure mathematics contexts is different from their application in the physics domain [1,24-29]. For instance, $\mathrm{Hu}$ and Rebello [11] exemplified how the use of integration differs in physics and mathematics. An understanding of integral as representing the Riemann sum suffices to tackle mathematics problems. However, in physics, students also need to understand the underlying meaning of the differential terms used for the physical system under consideration. As outlined by Redish [1], the mathematical expressions and the symbols comprising the expressions are used to represent physical systems and have conceptual meanings. The symbols express ideas and have units.

\section{A. Synthesis physics problems}

Most of the previous work highlighted in the earlier section has utilized single-concept problems. Although a 


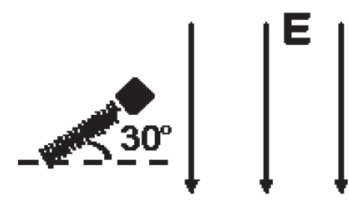

A uniform electric field of $8.6 \mathrm{kV} / \mathrm{m}$ is directed as shown. A projectile of mass $5 \mathrm{~g}$ and $+1 \mathrm{mC}$ charge is just outside the region, attached to a spring-based launcher. The spring, which has a spring constant $1 \mathrm{kN} / \mathrm{m}$ is compressed $10 \mathrm{~cm}$ and released. The projectile is launched into the region of the uniform electric field at an angle of $30^{\circ}$ to the horizontal. Ignore gravity. At some point, the projectile will be at the same vertical position as when it entered the field. What is the horizontal displacement of the projectile at that point?

(a)

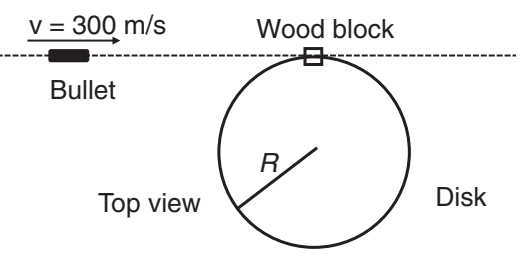

Tom shoots a bullet of mass $\mathrm{m}=0.02 \mathrm{~kg}$ and initial velocity $v=300 \mathrm{~m} / \mathrm{s}$ at a wood block attached to the outer rim of a disk, which in turn rests on a horizontal frictionless air table. The bullet rips through the block, emerging in the same direction, but at a lower speed of $100 \mathrm{~m} / \mathrm{s}$. Tom sees that the mass of the wood block can be ignored, so that the disk has a moment of inertia, with $M=1 \mathrm{~kg}$ and $R=25 \mathrm{~cm}$. (a) In what direction does the disk move after the collision? (b) What is the total kinetic energy of the disk after the collision?

(b)

FIG. 1. Two types of synthesis problem: (a) sequential and (b) simultaneous.

few studies on problem solving have used problems merging two or more physics concepts [30-33], there is a dearth of literature explicitly documenting how university students solve such problems. Moreover, little is known about students' use of mathematics and their difficulties when tackling physics problems merging multiple concepts that are more challenging.

Thus, for our study, we used quantitative synthesis problems, i.e., tasks consisting of two or more distinct concepts, typically from different chapters and separated in the teaching timeline [34]. The basis of synthesis problems is the merging of multiple concepts and equations during the concept application stage in order to build a solution. Further, we considered the two common types of synthesis problems, namely, sequential and simultaneous synthesis tasks. Figure 1 presents an illustration of a sequential synthesis problem and a simultaneous synthesis one [34].

The sequential synthesis problem is depicted in Fig. 1(a). The situation presents two events that can be analyzed in chronological order. A charged projectile is initially launched from a compressed spring. The projectile then undergoes a trajectory to enter a region of uniform electric field. Although these two events are parts of one situation and are connected by intermediate variables, it is possible to consider one event at a time. As a result, the two pertinent concepts (energy conservation and projectile motion) can be applied consecutively to solve the problem. Figure 1(b) is a simultaneous synthesis problem. The situation presents two events which occur concurrently. A disc is at rest on a frictionless surface. A bullet is fired on a wooden block attached to the disc causing a change in translational and rotational motion of the system simultaneously. These two events are such that they must be considered at the same time, thereby leading to the concurrent application of the two pertinent concepts (linear and angular momentum conservation).
Many earlier studies on single-concept problem solving have focused on the development of expertlike approaches among students by emphasizing qualitative task analysis, interpretation and understanding (see, e.g., Refs. [35-41]). In principle, these studies have used multistep problem solving strategies to encourage students to fully unpack the conceptual aspect of the task, and use qualitative depictions to mathematically express the concepts. They have additionally emphasized the key role of conceptual reasoning when dealing with mathematics, particularly for constructing the mathematical formulations and evaluating the quantitative solution. Further, the importance of conceptual reasoning while manipulating the equations has also been advocated [4]. In our study, we use quantitative synthesis problems which may emphasize qualitative analysis by requiring students to focus on the underlying concepts [34,42-44]. Further, these problems may not be easily solved by the formulaic plug-andchug approach. Synthesis problems require the blending of equations formulated from the application of multiple concepts. Apart from understanding the concepts underlying the individual equations, students also need to comprehend how the different mathematical expressions are connected. Synthesis problems may thus encourage solvers to engage in conceptual reasoning when manipulating equations, specifically when formulating and combining the multiple equations.

A previous study on quantitative synthesis problems investigated the role of guided conceptual scaffolding for promoting expertlike problem-solving approaches among students [34]. However, the majority of the students failed to find the correct quantitative solution despite the conceptual scaffolding. Ding et al. hypothesized that this may be due to the students' inability to combine the multiple concepts and/ or equations. A follow-up study [42] showed that mastery of single concept is a necessary but insufficient component for successfully solving synthesis problems. The students have 
difficulties identifying and applying the pertinent concepts. These initial studies have led us to propose potential bottlenecks to quantitative synthesis problems: (i) single-concept proficiency (mastery of applying individual concepts when solving a synthesis problem), (ii) concept identification (ability to identify all the pertinent concepts underlying the synthesis problem), (iii) joint application of concepts (proficient at applying multiple concepts in concert to solve the synthesis problem), and (iv) joint application of mathematical equations (proficient at manipulating the multiple equations in concert to solve the synthesis problem).

For the current study, we explored the effect of the mathematical complexity of the problem as a possible key factor in the bottlenecks. Particularly, we are interested in examining how mathematical complexity affects students' manipulation of mathematics, mainly their joint application of equations. In a separate study, we have focused on the conceptual aspect of synthesis problems where we report the effect of mathematical complexity on two bottlenecks, namely, the identification and the application of pertinent concepts [45].

\section{B. Mathematical complexity}

The mathematical complexity of a solution may depend on the mathematical structure in which the variable to be determined is imbedded in a problem. In the context of algebra, a single unknown problem posed as a "double reference" problem [e.g., $x-0.3 x=7$ ] is more complicated than a problem posed as a "single reference" [e.g., $(x-1) / 3=4]$ [46]. Additionally, a "start unknown" story problem [e.g., $(x-66) / 7=23]$ can be more complex than a "result unknown" problem [e.g., $2.65 \times 6+63=x$ ] [47]. For more complicated multistep problems, there is a composition effect [48]. Heffernan and Koedinger [48] found that students' performance on "composed" problems, which they defined as tasks combining two subproblems, thus requiring a merge of two steps and mathematical operations, was significantly worse than their aggregated performance on two matched single-step problems.

Here, we operationally define mathematical complexity as the number and the type of equations to be solved simultaneously due to the number of unknowns in each equation. Consider a problem with two unknowns and two equations. It is mathematically more difficult to concurrently manipulate two nonlinear equations (such as quadratic or trigonometric) each with two unknowns than to manipulate two linear equations, each with one unknown. In this study, the physics problems themselves do not change, rather the variables (knowns and unknowns) are manipulated such that the mathematical complexity is varied.

\section{Mathematical complexity and its influence on student mathematical performance}

In solving quantitative synthesis problems, mathematical complexity may interfere with the students' mathematical performance. We refer to mathematical performance as consisting of three aspects: formulation, combination, and simplification of mathematical equations. In our study, we propose that the formulation and combination of equations involve the blending of mathematical manipulation and conceptual reasoning. During these processes, students need to identify the relevant concepts and think about how to apply them in a situation comprising two or more events. Moreover, they are required to reflect on how the occurrence of one event is connected to another, how the multiple concepts are linked through intermediate variables and, hence, how the multiple equations are related. After these processes, the simplification of equations to determine the variable of interest requires the manipulation of equations as computational tools. It mostly requires identifying and canceling out known and unknown variables and rearranging the equation such that the required variable is written as a function of the others.

Mathematical complexity represents a potential difficulty for quantitative synthesis problems as follows. The mathematical complexity of a task can increase if (i) the number of unknowns in each equation increases, hence, leading to an increase in the number of steps to find each unknown, (ii) the complexity of the solution process increases (for example, manipulating quadratic or trigonometric equations), and (iii) both conditions highlighted earlier occur. This increase in mathematical complexity may make it difficult for students to comprehend how the multiple concepts are related in the given situation. Thus, students may struggle to identify the connecting variables and understand how to link the multiple equations. It may also negatively impact the students' fluency to combine the individual equations and simplify the resulting equation for the required variable. Previous studies have reported that students struggle with transferring and using their mathematical knowledge and skills in the physics context $[1,24,25,28]$. In our case, as mathematical complexity increases, the students may find it even more difficult to apply their mathematical knowledge in the physics domain.

Our study differs from previous ones that explore students' use of mathematics during physics problem solving in four ways. First, earlier studies have utilized single-concept problems [2,3,5-18]. In our study, we used quantitative synthesis (sequential and simultaneous) problems. These problems involve not only a combination of concepts but also the merging of mathematical equations after applying the concepts. Second, these equations do not include sophisticated mathematical operations such as integrals or derivatives. Instead, students have to manipulate algebraic expressions. At the university level, few studies have been implemented to explore students' use of algebra during physics problem solving [14,25]. Third, we incorporate conceptual reasoning during two stages of mathematical manipulation, namely, formulation and combination of equations. Last, the tasks are presented with 
varying levels of mathematical complexity from simple and linear equations to more intricate quadratic or trigonometric equations without changing the underlying physics.

We investigate the following overarching research question: How does mathematical complexity influence students' mathematical performance when solving quantitative sequential and simultaneous synthesis physics problems? We explore the effects of mathematical complexity on students' abilities to mathematically express pertinent concepts, combine multiple mathematical expressions, and simplify the equations to determine the variable of interest. We also explore possible reasons for similarities and differences in student mathematical performance on the sequential and simultaneous synthesis tasks.

In Sec. II we discuss the research design and data analysis. Section III presents the outcomes. The conclusions and discussions are highlighted in Sec. IV.

\section{METHOD}

The sequential synthesis problems were administered to 105 students and the simultaneous synthesis tasks were completed by 74 students. These students were enrolled in a second year mechanics course for physics majors. They completed the sequential and simultaneous synthesis tasks after studying the relevant concepts in their lectures. The mechanics course was traditionally taught, and the students worked in groups of 3-4 during recitation to practice problem solving. They were not explicitly taught how to solve synthesis problems. However, they had opportunities to solve multiconcept problems as homework and in class. They were also provided with the solutions to these problems. Grading of the synthesis problems focused on the application of pertinent concepts for setting up equations, on the combination of pertinent equations, and on the final answer obtained.

\section{A. Tasks}

The final sets of the sequential (shown in Fig. 2) and simultaneous (shown in Fig. 4) synthesis problems were obtained after being vetted with a group of students, physics education researchers, and physicists. In particular, we checked that the students did not misinterpret the problem statement and the quantitative information used is rational. We also ensured that the problems are based on topics which the students have learned in class.

\section{Sequential synthesis problems}

The sequential synthesis problems are presented in Fig. 2. We designed three versions of the same problem, with varying mathematical complexity, namely, simple, intermediate, and complex. Each task depicts a block on a ramp angled at $\theta$ being propelled from a spring. The block then undergoes a trajectory and lands on another surface inclined at $\phi$. The two pertinent concepts for tackling the three versions of the sequential task are energy conservation and projectile motion.

Four fundamental equations (as shown in Fig. 3) are required to solve all three versions of the problem. Application of energy conservation results in Eq. (1) connecting the initial energy of the system (spring potential energy) to its final energy (block's kinetic energy and gravitational potential energy). Application of projectile motion leads to three equations: Eq. (2) and Eq. (3) for the horizontal and vertical motion respectively, and Eq. (4), trigonometry relating the horizontal and vertical motions.

Simple: An apparatus uses a spring launcher to fire a block (mass $2 \mathrm{~kg}$ ) over a ridge. The spring (spring constant, $\mathrm{k}=3000 \mathrm{~N} / \mathrm{m}$ ) is compressed $0.1 \mathrm{~m}$ and releases the block from its equilibrium position, which is located a distance $L=0.5 \mathrm{~m}$ from the crest. If the angles shown are $\theta=35^{\circ}$ and $\phi=25^{\circ}$, what is the horizontal distance $R$ from the crest to where the block hits the slope on the other side?

Intermediate: An apparatus uses a spring launcher to fire a block (mass $2 \mathrm{~kg}$ ) over a ridge. The spring (spring constant, $\mathrm{k}=3000 \mathrm{~N} / \mathrm{m}$ ) is compressed to an unknown distance $x$, and releases the block from its equilibrium position, which is located a distance $L=0.5 \mathrm{~m}$ from the crest. The block lands on the opposite slope, a horizontal distance $R=1.3 \mathrm{~m}$ from the crest. If the angles shown are $\theta=35^{\circ}$ and $\phi=25^{\circ}$, what was the initial compression of the spring, $x$ ?

Complex: An apparatus uses a spring launcher to fire a block (mass 2kg) over a ridge. The spring (spring constant, $\mathrm{k}=3000 \mathrm{~N} / \mathrm{m}$ ) is compressed $0.1 \mathrm{~m}$ and releases the block from its equilibrium position, which is located a distance $L=0.5 \mathrm{~m}$ from the crest. The block lands on the opposite side, a horizontal distance $R=1.3 \mathrm{~m}$ from the crest. If $\phi=25^{\circ}$, what was the angle of the initial slope, $\theta$ ?

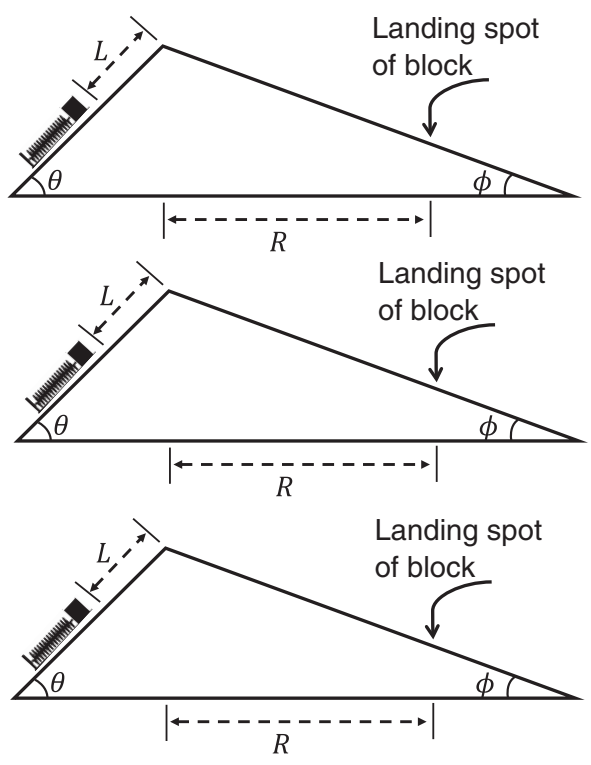

FIG. 2. The three versions of the sequential synthesis problems. 
Energy conservation: $1 / 2 \mathrm{k} x^{2}=1 / 2 \mathrm{~m} v^{2}+\mathrm{m} g(L+x) \sin \theta \ldots .(1)$

Projectile motion: $R=v \cos \theta t$

$y=v \sin \theta t-1 / 2 \mathrm{~g} t^{2}$

$\tan \phi=-y / R$.

FIG. 3. The four essential equations for tackling the sequential synthesis problems.

Table I presents information about the known and unknown variables and the types of equations to be manipulated for the simple, intermediate, and complex version of the sequential problem. For the simple task, $v$ could be determined at the onset from Eq. (1) and, hence, could be regarded as a known in Eqs. (2) and (3). For the intermediate and complex tasks, a value for $y$ could be solved at the onset from Eq. (4) and could be considered as a known in Eq. (3).

\section{Simultaneous synthesis problems}

As for the simultaneous synthesis problems (shown in Fig. 4), we designed two versions, namely, simple and complex involving the same physics but with varying mathematical complexity. They are both based on the situation of a disc sliding on a frictionless surface while a projectile is released by a spring launcher. When the compressed spring releases the projectile the disc spins, resulting in energy transfer and a change in translational and rotational motion of the system. Energy conservation and linear and angular momentum conservation are the pertinent concepts for tackling both versions of the problem.

Three main equations, depicted in Fig. 5, are required for solving both tasks.

Application of energy conservation results in Eq. (1) connecting the different types of energy for the initial and final conditions. Application of linear momentum conservation gives Eq. (2) relating the initial momentum of the system to its final momentum. Application of angular momentum conservation results in Eq. (3) connecting the initial angular momentum of the system to its final angular momentum. Information about the known and unknown variables and the types of equations to be manipulated for the simple and complex version of the simultaneous synthesis task is shown in Table II. For the simple task, $v_{f}$ and $\omega$ can be obtained at the onset from Eq. (2) and Eq. (3), respectively. Hence, these variables were treated as knowns in Eq. (1). "Types of equations" refers to the final equation obtained, from which the required variable ( $\Delta x$ or $v_{o}$ ) can be solved for.

\section{B. Administration of sequential and simultaneous synthesis problems}

Data were collected from written tasks and individual interviews during the "flexible homework" assignment [49]. It is a $1 \mathrm{~h}$ session where students engage in activities such as tutorials and interviews as a replacement for one of their homework assignments. The students earn full credits for participating in the "flexible homework".

A total of 105 students completed the sequential synthesis tasks. Ninety-two of them signed up to complete the written tasks only. The remaining 13 students signed up for a $1 \mathrm{~h}$ individual interview session. For those who completed the written tasks, each student was randomly given one version of the problem, simple $(n=30)$, intermediate ( $n=31)$, and complex $(n=31)$. Across the three groups, the students had similar level of average course grade $[F(2,91)=0.127, \quad p=0.881]$. They completed the given task on their own, without consulting their peers. Irrespective of the task's mathematical complexity, they were given approximately $30 \mathrm{~min}$ to tackle the problem.

TABLE I. Known and unknown variables, and types of equations across sequential synthesis tasks with increasing mathematical complexity ( $m$, mass of block; $k$, spring constant; $L$, equilibrium position; $\phi$, angle of final slope; $x$, initial compression of spring; $\theta$, angle of initial slope; $R$, horizontal distance; $v$, block's velocity at crest of the ramp; $y$, block's final vertical position; $t$, time taken by block to hit the landing spot). Types of equations refer to the final equation obtained after manipulation, from which a value for the unknown variable of interest $(R, x$, and $\theta)$ can be found. See Appendix A for the solutions.

\begin{tabular}{|c|c|c|c|c|}
\hline & & Simple task & Intermediate task & Complex task \\
\hline Common given information & & \multicolumn{3}{|c|}{$m, k, L$, and $\phi$} \\
\hline Additional given information & & $x$ and $\theta$ & $R$ and $\theta$ & $R$ and $x$ \\
\hline Variable to solve for & & $R$ & $x$ & $\theta$ \\
\hline $\begin{array}{l}\text { Unknowns in each equation: } \\
\frac{1}{2} k x^{2}=\frac{1}{2} m v^{2}+m g(L+x) \sin \theta \\
\qquad \begin{array}{l}R=v \cos \theta t \\
y=v \sin \theta t-\frac{1}{2} \mathrm{~g} t^{2} .\end{array} \\
\quad \tan \phi=\frac{-y}{R}\end{array}$ & $\begin{array}{l}(1) \\
(2) \\
(3) \\
(4)\end{array}$ & $\begin{array}{c}v \\
R, t \\
y, t \\
y, R\end{array}$ & $\begin{array}{l}v, x \\
v, t \\
v, t \\
y\end{array}$ & $\begin{array}{l}v, \theta \\
v, \theta, t \\
v, \theta, t \\
y\end{array}$ \\
\hline Types of equations & & Nonquadratic & Quadratic & Quadratic with trigonometry embedded \\
\hline
\end{tabular}


Simple: A disc of mass $3.0 \mathrm{~kg}$ and radius $R=0.75 \mathrm{~m}$ is sliding (face down) at velocity $v_{o}$ along a frictionless surface. A spring launcher is fixed outside the edge of the disc. The spring (spring constant, $\mathrm{k}=49.0 \mathrm{kN} / \mathrm{m}$ ) is compressed a distance $\Delta x$ and releases a projectile of mass $6.0 \mathrm{~kg}$ from its equilibrium position. After release, the velocity of the projectile relative to the surface is $v_{p}=11.0 \mathrm{~m} / \mathrm{s}$. Assume that the mass of the launcher is small and that the spring releases the projectile very rapidly. If the initial velocity of the disc was $v_{0}=5.0 \mathrm{~m} / \mathrm{s}$, what was the initial compression of the spring, $\Delta x$ ?

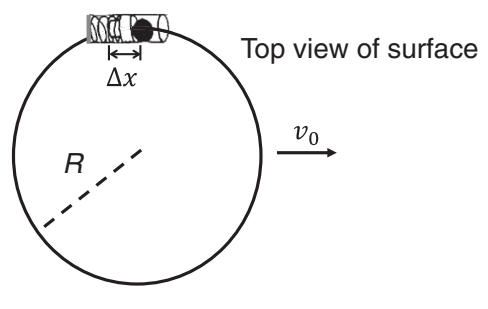

Complex: A disc of mass $3.0 \mathrm{~kg}$ and radius $R=0.75 \mathrm{~m}$ is sliding (face down) at velocity $v_{o}$ along a frictionless surface. A spring launcher is fixed outside the edge of the disc. The spring (spring constant, $\mathrm{k}=49.0 \mathrm{kN} / \mathrm{m}$ ) is compressed a distance $\Delta x$ and releases a projectile of mass $6.0 \mathrm{~kg}$ from its equilibrium position. After release, the velocity of the projectile relative to the surface is $v_{p}=11.0 \mathrm{~m} / \mathrm{s}$. Assume that the mass of the launcher is small and that the spring releases the projectile very rapidly. If the initial compression of the spring was $\Delta x=17.5 \mathrm{~cm}$, what was the initial velocity of the disc, $v_{o}$ ?

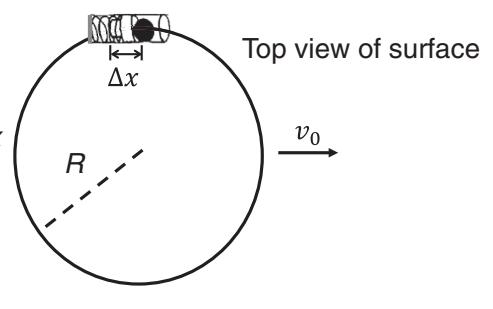

FIG. 4. The two versions of the simultaneous synthesis problem.

$$
\begin{aligned}
& \text { Energy conservation: } 1 / 2 \mathrm{k} \Delta x^{2}+1 / 2(\mathrm{M}+\mathrm{m}) v_{o}^{2}=1 / 2 m v_{p}^{2}+1 / 2 M v_{f}^{2}+1 / 2 \mathrm{l} \omega^{2} \ldots . .(1) \\
& \text { Linear momentum conservation: }(\mathrm{M}+\mathrm{m}) v_{o}=M v_{f}+m v_{p} \\
& \text { Angular momentum conservation: } \mathrm{m} v_{o} R=\mathrm{m} v_{p} R+\mathrm{I} \omega
\end{aligned}
$$

FIG. 5. The three essential equations for solving the simultaneous synthesis problems.

Each of the 13 students in the interviews was randomly assigned one version of the problem, simple $(n=4)$, intermediate $(n=5)$, and complex $(n=4)$. In each session, the student was given $30 \mathrm{~min}$ to solve the problem followed by an interview session of approximately $30 \mathrm{~min}$.
The same process was used for administering the written tasks and individual interviews to the 74 students who completed the simultaneous synthesis problems. Sixty-six students signed up to complete the written tasks only. The remaining 8 students signed up for a $1 \mathrm{~h}$ individual

TABLE II. Known and unknown variables, and types of equations across simultaneous synthesis tasks with increasing mathematical complexity $\left(M\right.$, mass of disc; $R$, radius of disc; $v_{o}$, initial velocity of disc; $v_{f}$, final velocity

\begin{tabular}{|c|c|c|c|}
\hline & & Simple task & Complex task \\
\hline Common given information & & \multicolumn{2}{|c|}{$M, m, R, k$, and $v_{p}$} \\
\hline Additional given information & & $v_{o}$ & $\Delta x$ \\
\hline Variable to solve for & & $\Delta x$ & $v_{o}$ \\
\hline $\begin{array}{l}\text { Unknowns in each equation: } \\
\begin{aligned} \frac{1}{2} k \Delta x^{2}+\frac{1}{2}(M+m) v_{o}^{2} & =\frac{1}{2} m v_{p}^{2}+\frac{1}{2} M v_{f}^{2}+\frac{1}{2} I \omega^{2} . \\
(M+m) v_{o} & =M v_{f}+m v_{p} . \\
m v_{o} R & =m v_{p} R+I \omega\end{aligned}\end{array}$ & $\begin{array}{l}(1) \\
(2) \\
(3)\end{array}$ & $\begin{array}{l}\Delta x \\
v_{f} \\
\omega\end{array}$ & $\begin{array}{c}v_{o}, v_{f}, \omega \\
v_{o}, v_{f} \\
v_{o}, \omega\end{array}$ \\
\hline Types of equations & & $\begin{array}{c}\text { Independent quadratic } \\
\text { equation. }\end{array}$ & $\begin{array}{l}\text { Quadratic equation embedded } \\
\text { in a system of } 2 \text { equations. }\end{array}$ \\
\hline
\end{tabular}
of disc; $\omega$, angular velocity of disc; $m$, mass of projectile; $v_{p}$, final velocity of projectile; $k$, spring constant; $\Delta x$, initial compression of spring). See Appendix B for the solutions. 
interview session. For the written tasks, the students were randomly assigned one version of the problem (simple, $n=33$; complex, $n=33$ ). In this case, there was no significant between-group difference indicating that the students were at the same grade level $[F(1,65)=0.242$, $p=0.625]$. All the students were given approximately $30 \mathrm{~min}$ to handle the problem. For the interviews, each of the 8 students was randomly assigned one version of the problem ( $n=4$ for simple task; $n=4$ for complex task). Each student was first allocated $30 \mathrm{~min}$ to complete the task. They were then immediately interviewed for a maximum of approximately $30 \mathrm{~min}$.

\section{Analysis of sequential and simultaneous synthesis problems}

From the written solutions to the sequential and simultaneous synthesis problems, the students' mathematical performance was analyzed in three levels, namely, formulation, combination, and simplification of equations.

For formulation of equations, we considered if the students identified all the pertinent concepts underlying the task and generated all the equations to mathematically express the identified concepts. For combination of equations, we looked at whether or not the students combined their formulated equations associated with the pertinent concepts. For simplification of equations we coded the students' attempt to identify variables and rearrange equations such that the required variable is written as a function of the others. The detailed coding scheme for the sequential and simultaneous synthesis problems is presented in Appendixes $\mathrm{C}$ and $\mathrm{D}$, respectively. The coding of the students' written solutions was done independently by two researchers. For the sequential synthesis problems, an interreliability rate of $87 \%$ was obtained. For the simultaneous synthesis problem, the intercoder agreement was $88 \%$. After discussion, all the differences were resolved. We conducted $\chi^{2}$ tests at the three analysis levels to detect any differences across the synthesis tasks with varying mathematical complexity.

From the written solutions, we additionally explored why the students did not combine and simplify the equations in the sequential and the simultaneous synthesis problems. We examined their actions by focusing on whether they merely wrote down the steps to find the variable of interest or whether they executed the steps. We also considered if the students dealt with linear, quadratic, or trigonometric equations in a purely symbolic form or if they immediately substituted values as they generated the expressions. Further, we looked at how the students applied their mathematical knowledge, such as trigonometric identity, factorization, and expansion of algebraic expressions to the physics problems.

We have two main themes extracted from the individual interviews. They were analyzed thematically for the link between the students' mathematical performance, their approach to tackle the two types of synthesis problems (if they divided the situation into segments or treated it as a single event), and their unpacking of the physical situations (if they realized there was more than one event in the situation, understood how the occurrence of one event was connected to another; hence, how the different concepts were related). We also examined the interviews for possible reasons why students failed to combine and simplify the equations after formulating them for the two types of synthesis problems. The interview protocol is in Appendix E.

\section{RESULTS}

We first present the findings on the effects of mathematical complexity on students' mathematical performance on the sequential and simultaneous synthesis problems. We then report the reasons why students did not combine and simplify their equations for the two synthesis problem types. We also present the outcomes on the link between the students' mathematical performance, their approaches to tackle the situation in the sequential and simultaneous problems, and their unpacking of the physical situations.

\section{A. Written results}

Figure 6 presents the percentage of students who properly formulated all the pertinent equations, combined the equations, and simplified the combined equations for the two types of synthesis problems with different mathematical complexity. For instance, if a student formulated all the relevant equations, combined them but did not successfully simplify the combined equations, then the student was classified within the "formulate" and the "combine" category, respectively, but not in the "simplify" category.

For the sequential synthesis tasks, Fig. 6(a), there was no significant difference across the three conditions in the number of students who formulated the pertinent equations $\left(\chi^{2}=0.280, p=0.869\right)$ and combined these equations $\left(\chi^{2}=0.142, p=0.931\right)$. However, we noted a significant difference across the three tasks in the number of students who attempted to simplify the equations to determine the required variable $\left(\chi^{2}=8.20, p=0.017\right)$. Thus, for the sequential synthesis problems, mathematical complexity seemed to impact students' mathematical performance only when simplifying the equation to obtain the variable of interest.

For the simultaneous synthesis tasks, Fig. 6(b), there was a substantial between-group difference at all the three analysis levels (formulation of equations: $\chi^{2}=5.28, p=$ 0.022; combination of equations: $\chi^{2}=5.80, p=0.016$; simplification of equation: $\left.\chi^{2}=5.80, p=0.016\right)$. This indicates that mathematical complexity may have influenced the students' formulation and combination of 


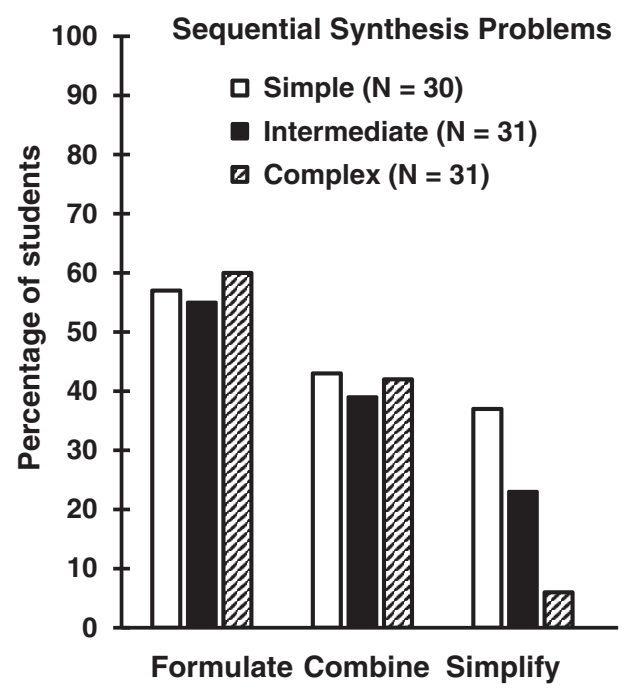

(a)

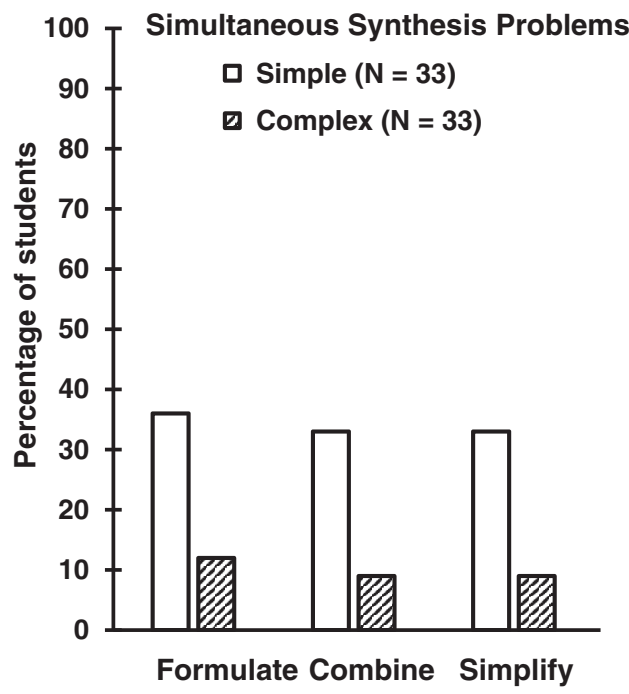

(b)

FIG. 6. Mathematical performance on synthesis tasks with varying mathematical complexity: (a) sequential and (b) simultaneous synthesis task.

equations as well as simplification of equations to determine the required variable for the simultaneous synthesis problems.

Overall, as the task's mathematical complexity increases, the students' mathematical performance on the sequential and simultaneous synthesis tasks decreases. For the sequential tasks, mathematical complexity appears to negatively influence the students' mathematical performance only at the stage of simplifying the equation for the required variable. For the simultaneous tasks, mathematical complexity has a negative effect across all the three stages.

We made a comparison between the two types of synthesis problem for the number of students who formulated all the fundamental equations. We also compared the number of students who properly combined the pertinent equations among those who formulated them, and the number of students who simplified the equations among those who combined them. The outcomes, for the sequential and simultaneous synthesis tasks with varying mathematical complexity, are shown in Fig. 7.

Regardless of the task's mathematical complexity, the students were better able to formulate all the pertinent equations for the sequential synthesis tasks (simple, 57\%; intermediate, $55 \%$; complex, $61 \%$ ) as opposed to the simultaneous tasks (simple, 36\%; complex, 12\%). This also shows that the students were more successful in identifying all the pertinent concepts for the sequential tasks compared to the simultaneous problems. In order to formulate all the fundamental equations, the students need to first identify all the pertinent concepts. However, after
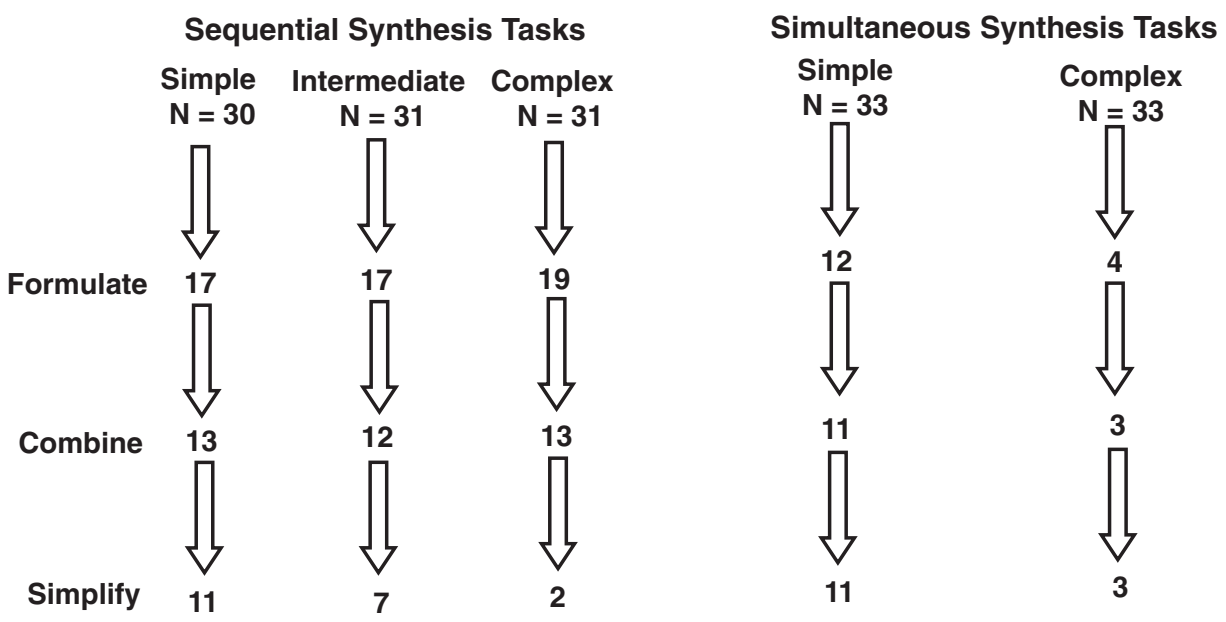

FIG. 7. Number of students formulating all the fundamental equations, combining the formulated equations and simplifying the combined equations for the sequential and simultaneous synthesis tasks with varying mathematical complexity. 
formulating the pertinent equations, the students were more successful in combining them for the simultaneous tasks (11 out of 12 students, equivalent to $92 \%$, for the simple group, and 3 out of 4 students, equivalent to $75 \%$, for the complex group) as opposed to the sequential tasks (13 out of 17 students, equivalent to $76 \%$, for the simple group, 12 out of 17 students, equivalent to $71 \%$, for the intermediate group, and 13 out of 19 students, equivalent to $68 \%$, for the complex group). They were also better able to simplify the combined equations for the simultaneous tasks (11 out of 11 students, $100 \%$, for the simple group, and 3 out of 3 students, $100 \%$, for the complex group) compared to the sequential problems (11 out of 13 students, equivalent to $85 \%$, for the simple group, 7 out of 12 students, equivalent to $58 \%$, for the intermediate group, and 2 out of 13 students, equivalent to $15 \%$, for the complex group).

We considered the student's actions (written solutions) to explore why they did not combine and simplify the equations after formulating them. We found two main reasons. One related to the features of the equations, and the other pertains to the students' ability to apply their mathematical knowledge of trigonometric identity, factorization and expansion of algebraic expressions to the physics problems. For the sequential synthesis tasks, the students manipulated quadratic equations, involving trigonometry, which became more complicated for the intermediate and complex tasks, and when left in a purely symbolic form. They failed to apply their mathematical knowledge of trigonometric identity when solving the complex problem. For the simultaneous synthesis tasks, the students dealt with linear expressions and quadratic equations with no trigonometry involved. Regardless of mathematical complexity, these equations were relatively simple to manipulate compared to those in the sequential tasks. The students were able to apply their mathematical knowledge of factorization and expansion of algebraic expressions.

We first looked at the actions of those students who did not combine the pertinent equations after formulating them for the sequential synthesis tasks. The outcomes and examples of students' actions for the simple, intermediate and complex sequential synthesis tasks are in Appendix F. The prominent outcome is that the students knew at a conceptual level how to obtain the necessary intermediate variables and the final variable of interest from the pertinent equations. We observed that some students drew an arrow linking the energy conservation equation to the expression obtained after merging the projectile motion equations. We also noted that the students re-arranged the energy conservation equation with velocity as the key variable and determined an expression for time from the projectile motion equations. Further, after formulating the fundamental equations, the students wrote down the steps to find the intermediate variables and final variable of interest from these equations. However, they failed to combine the energy conservation equation with the projectile motion equations. This is because the equations became more complicated to manipulate, mainly for the intermediate and complex tasks. The students tended to leave the equations in symbolic forms, which increased the complexity of the expressions. Further, after merging the projectile motion equations, an intricate quadratic equation with trigonometry emerged. The fact that the equations were complicated might have made it difficult for the students to combine the resulting projectile motion equation with the energy conservation equation. In one case of the simple task, the student seemed to spend a lot of time trying to solve the problem using an inappropriate concept, Newton's second law. The student then shifted to using energy conservation and projectile motion but ran out of time to solve the problem using these pertinent concepts and equations.

We also explored why the students did not simplify their equations after combining them for the sequential synthesis tasks. The results and illustrations of students' actions for the simple, intermediate and complex sequential synthesis tasks are in Appendix G. Overall, the main outcome is that regardless of the task's mathematical complexity, after combining the energy conservation and projectile motion equations, the students arrived at quadratic expressions involving trigonometry, which they usually left in a purely symbolic form. Although they were given the values for certain variables, these were not substituted, and therefore the quadratic expressions became increasingly complicated. Moreover, for the complex sequential synthesis task, the students had to use their mathematical knowledge of trigonometric identity which they failed to apply.

Overall, for the sequential synthesis tasks, the students manipulated quadratic expressions with trigonometry which became more intricate for the intermediate and complex tasks. The complexity of the equations increased if these equations were left in symbolic forms and after the projectile motion equations were merged. The students also failed to apply their mathematical knowledge of trigonometric identity. As a result, they did not combine and simplify their equations.

For each of the simple and complex simultaneous tasks, all but one student attempted to combine and simplify their formulated equations. Although the exact mechanism for such a failure was unclear from the written solutions, we observed some consistent patterns among those who successfully combined and simplified the formulated equations. All the students who combined the formulated equations proceeded with simplifying the expression to find a value.

In this case, the students formulated one quadratic equation (energy conservation) and two linear equations (angular and linear momentum conservation). For the simple task, the students found a value from each of the linear equations which they plugged into the quadratic equation. For the complex task, they substituted their linear 
expressions in the quadratic equation. The handling of values or linear expressions seemed easy and straightforward for the students, which facilitated their attempt to combine the equations. Once combined, a quadratic equation emerged. It was straightforward to manipulate for the simple task, which dealt mainly with numbers. For the complex task, however, the students had to use their mathematical knowledge of factorization and expansion of algebraic expressions, which they successfully applied. The results and examples of students' actions for the simple and complex simultaneous tasks are in Appendix $\mathrm{H}$.

Hence, for the simultaneous synthesis tasks, regardless of mathematical complexity, the students properly combined and simplified the equations as they manipulated the linear and quadratic expressions. They were able to apply their mathematical knowledge of factorization and expansion of algebraic expressions.

\section{B. Interview results}

From the individual interviews we first explored the connection between the students' mathematical performance, how they approached the situation in the sequential and simultaneous synthesis problems and their unpacking of the physical situations.

For the sequential synthesis problems, the most prominent outcome is that irrespective of the task's mathematical complexity, all the students split the physical situation into segments. They realized there were more than one event and displayed an understanding of the link between the events. They identified all the pertinent concepts which they expressed mathematically. Further, they recognized all the connecting variables, demonstrated a comprehension of how to combine their equations and use the intermediate variables to obtain the final variable of interest. Typical interview responses for students who completed the simple, intermediate, and complex version of the problem are shown in Fig. 8.

In the examples of students' responses, irrespective of the sequential task's mathematical complexity, the students divided the situation into segments and recognized that it comprised two events. This is indicated by their use of the verbal expressions "first part or second part of the problem" and "broke this problem into two". They also identified the two pertinent concepts, energy conservation and projectile motion, and mathematically expressed each concept. Further, they recognized velocity and time as the necessary intermediate variables for connecting the events and hence the pertinent concepts. A value or expression for the velocity of the block at the crest of the ramp was obtained from the energy conservation or projectile motion equations. A value or expression for the time taken by the block to hit the landing spot was determined from the projectile motion equations. The students also explained how they would solve for the final variable of interest by combining their values or expressions for velocity and time from the energy conservation and projectile motion equations.

For the simultaneous synthesis problems, the prominent outcome is that the majority of the students who completed the complex version of the task ( 3 out of 4 students) treated the physical situation as a single event and used one concept for problem solving. As such they formulated only one equation, for the one pertinent concept identified, and did not combine any equations. The majority of the students who completed the simple version of the problem ( 3 out of 4 students) realized there was more than one event. However, they recognized part of the multiple events, instead of all of them, thus identified part of the multiple pertinent concepts which they expressed mathematically. Further, they combined the formulated equations and only identified part of the necessary connecting variables to solve the problem. Regardless of the task's mathematical

\begin{abstract}
Student RP (simple task): "The problem is asking for the distance from the tip of the crest which is not difficult to find altogether. I decided to think through the first part of the problem with energy conservation to determine the velocity at the crest. For the second part, I use the velocity at the crest and projectile motion to find how long it takes for the block to travel through the air and hit the ground. From there I use time and the horizontal component velocity to find $R$ [horizontal distance]."
\end{abstract}

Student DM (intermediate task): "I started with the second part of the problem where I use projectile motion, set the equation for the $y$ distance travel [equation for vertical motion] and the $x$ distance [equation for horizontal motion]. From the $x$ equation, I can find the time that the object flies in the air. Once I get the time I can substitute it in the $y$ equation to get the velocity [of the object]. Then I use conservation of energy [...]. I can get an expression in terms of $x$ [initial compression of the spring] from it [energy conservation equation], substitute velocity from projectile motion and solve for $x$."

\footnotetext{
Student RV (complex task): "I broke this problem into two. Here is your first part where you have a spring potential energy becoming kinetic energy and also gravitational potential [energy] to give you the velocity of the block. From there it goes from conservation of energy equation to projectile motion equations where the second part is taking into account the trajectory the block is launched. I set my equation for horizontal and vertical motion. From the projectile motion equations I can get rid of time, time that the block is in the air, and plug in the velocity of the block to find the angle [angle of initial slope, $\theta$ "
}

FIG. 8. Examples of interview responses for the three versions of the sequential synthesis task. 


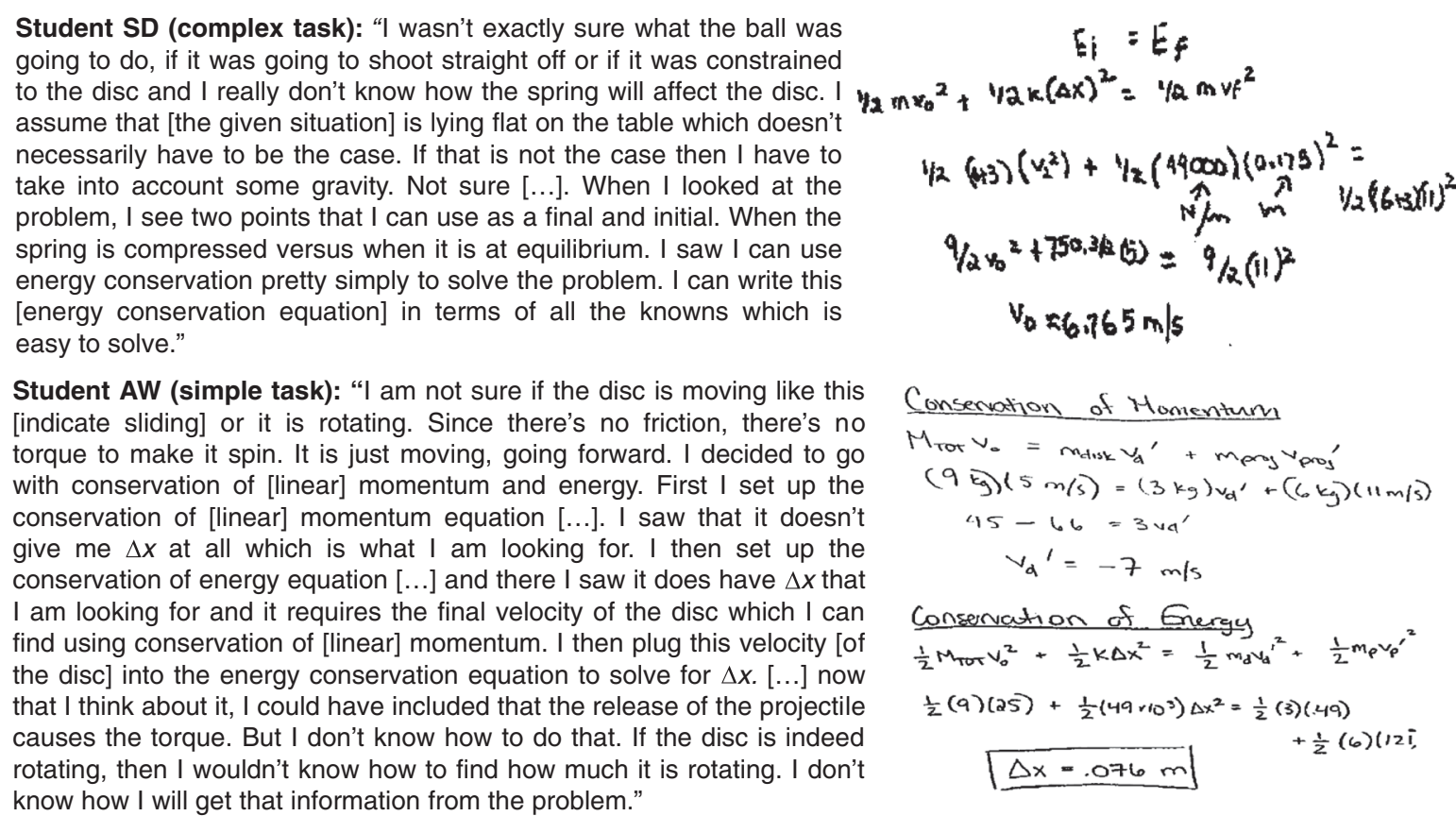

FIG. 9. Examples of interviews and written responses for the complex and simple simultaneous synthesis problems.

complexity, the students were not capable of fully unpacking the given situation and making predictions by connecting the task to learned physics concepts. Examples of the interview and written responses from the students completing the complex and simple versions of the problem are shown in Fig. 9.

The student who completed the complex version of the simultaneous task reduced a multiple events situation into a single event one comprising only energy conservation, typically when the spring is compressed and when it releases the projectile. As such the student used only one pertinent concept, energy conservation, and mathematically expressed this one identified concept. In this case, the need to combine multiple different equations does not arise as the student formulated one equation comprising all the known variables to determine the initial velocity of the disc which is the aim of the task.

The student who completed the simple version of the simultaneous task realized there were more than one event in the situation and identified two pertinent concepts, energy conservation and linear momentum conservation. As such, the student formulated two equations (one for linear momentum conservation and one for energy conservation) and combined them. Hence, the student identified only part of the intermediate variables, the final velocity of the disc, to solve the problem. A value for the final velocity of the disc was obtained from the linear momentum conservation equation. This value was then plugged into the energy conservation equation, a quadratic equation which can be easily simplified to find the variable of interest, the spring compression.
Overall, regardless of the mathematical complexity of the sequential synthesis task, the students divided the situation into segments and fully unpacked the physical situation which seemed to facilitate their formulation and combination of equations. They were also able to explain, at a conceptual level, how they would obtain the key connecting variables and the final variable of interest from the pertinent equations. In contrast, for the simultaneous synthesis problems, the students struggled to fully unpack the physical situation which appeared to impede their formulation and combination of equation. They treated the complex simultaneous task as a single event and formulated only one equation such that the need to combine equations did not arise.

From the individual interviews, we also investigated the reasons for students not combining and simplifying the fundamental equations after formulating them. Consistent with our analysis for the written solutions, for the interview responses we considered only students who identified all the pertinent concepts and formulated all the fundamental equations. As such, the outcomes reported here pertain only to the sequential synthesis tasks. The eight interviewees completing the simultaneous synthesis tasks either identified one pertinent concept thus formulating one equation (mainly for the complex version) or identified two pertinent concepts hence formulating two fundamental equations (mainly for the simple version). Of the 13 interviewees completing the sequential synthesis tasks, only four of them either did not combine or did not simplify the equations. Two of these students did not combine the equations after formulating them. They tackled either the simple or the 
Student SB (simple task, formulate all equations and do not combine): "I have to break this task into two separate tasks. The first task is to find how much velocity you are going to get in order to launch the block and then using this velocity to know where the block will land. [...] I have to equate energies to find out the velocity first. Then I use $R=v_{o} \cos \theta t$ to solve for $t$ which I will plug in the $y$ equation [equation for vertical motion]. You have to equate the $y$ equation to the slope of the line which is $-\tan \phi$ and then you just plug in values to find $R$. [...] the concepts are pretty straightforward. The algebra, I got mix up with so many symbols and signs, I can easily make an error. I got stuck here just because of the algebra which looks complicated and I have difficulty naming notations."

Student RC (complex task, formulate all equations and do not combine): "Conceptually I knew what's going on in the task but I don't know how to show that completely mathematically. It seems like I have too many variable and not enough information to solve it. I started by finding the velocity that this block will leave the spring with by using conservation of energy. And then I started writing down basic kinematics equations, your change in $x$ distance [horizontal motion equation] and your change in $y$ [vertical motion equation]. And then I set tan $\phi$ equal to $-y / R$. $R$ is $1.3 \mathrm{~m}$. Then I set $1.3=v_{o} \cos \theta t$. From all these [projectile motion equations] I can get rid of time. But then the equation looks complicated."

Student NC (intermediate task, combine equations and do not simplify): "This is kind of two problems because you have to solve for each thing separately. I started from the chronological beginning where the spring is compressed. I use conservation of energy to find the velocity of the block. I got an expression for $v$ that involves $x$. Then I use projectile motion, equations for horizontal and vertical motion to find the time the block will land. [...] I basically merge the two concepts to come up with this compression [of the spring]. If you know everything over here on this side [show the trajectory event], you can get a value for the velocity and then that can give you a value for the compression [of the spring]. [...] I think if I evaluated these numerically I would have reduced the algebra. My plan was to get an expression for the distance that it is compressed and then plug in all the values at the end. But I left them in the form of algebra which complicated things."

Student NY (complex task, combine equations and do not simplify): "I find the task difficult mathematics wise. It can be a lot simpler way to do it. [...] I divided the problem into two parts. This one [show the spring event] you have to find the velocity and then after that you simplify it as a projectile motion problem. You use energy conservation to find the velocity of the block and from there you use projectile motion to know where it lands. The issue with that is the mathematics become very ugly very quickly. It doesn't work out nicely. I use projectile motion, decompose my velocity into $x$ and $y$. I need to get how long the block is in the air from the equation for $y$ position and plug it in the expression for the $x$ distance since we know $R$ [horizontal distance]. I then plug the velocity expression and then from there I try to simplify for $\theta$. Unfortunately this is in terms of $\theta$ which will lead to mathematical difficulties. [...] The main difficulty here is just solving the math."

FIG. 10. Interview responses for students failing to combine the formulated equations, and those failing to simplify the combined equations for the sequential synthesis tasks.

complex task. The remaining two participants combined the equations but did not simplify them. They completed either the intermediate or the complex problem.

These four students mentioned that the equations are complicated with many signs, symbols, and variables which made it difficult for them to manipulate to find a quantitative solution. It must be pointed out that all the four students correctly articulated the physics underlying the task regardless of mathematical complexity. They were able to identify the necessary intermediate variables and explain how to obtain these and the final variable of interest from the pertinent equations. The interview responses are presented in Fig. 10.

Thus, for the sequential synthesis tasks, the interview results indicate that the students did not combine and simplify the equations because the mathematics was rather complicated, particularly when left in the symbolic form. However, the students were able to highlight, at a conceptual level, how they would obtain the connecting variables and the final variable of interest from the pertinent equations.

\section{CONCLUSIONS AND DISCUSSIONS}

This study indicates that mathematical complexity negatively influences the students' mathematical performance on the sequential and simultaneous synthesis problems. However, for the sequential synthesis tasks, mathematical complexity affects only the students' simplification of equation for obtaining the variable of interest. For the simultaneous synthesis tasks, it additionally affects the students' formulation and combination of equations which in a sense also relates to conceptual reasoning.

Further, the results from the individual interviews show that the students approached the sequential and simultaneous tasks differently. Their approaches seemed to influence their unpacking of the physical situation, which in turn interfered with their mathematical performance on formulating and combining the equations. The students divided the sequential tasks into a sequence of subtasks, regardless of their mathematical complexity. As a result, their unpacking of the physical situation was greatly facilitated and their formulation and combination of equations were mostly successful. For the simultaneous synthesis tasks, especially the complex version, the students oversimplified the situation as a single-concept event. Irrespective of the task's mathematical complexity, the students failed to fully unpack the situation, which may have hindered their formulation and combination of equations.

Sequential synthesis problems include the chronological occurrence of events. Even if the events are connected, it is possible to consider one event at a time which may have led the students to divide the situation into segments, as observed from the individual interviews, regardless of the task's mathematical complexity. Consequently, the students may have been prompted to consider each event individually, which facilitated their unpacking of the situation. As evident from the individual interviews, 
irrespective of the task's mathematical complexity, the students identified all the events, comprehended the link between them, identified all the pertinent concepts, and recognized all the key connecting variables. Hence, the students' formulation and combination of equations may have been favorably influenced. As noted from the interviews, the students mathematically expressed all the pertinent concepts. They were also able to explain, at a conceptual level, how they will obtain the necessary intermediate variables and the variable of interest from the fundamental equations. In this case, the characteristics of the sequential synthesis task seemed to be a dominant factor that influenced the students' approaches to and unpacking of the physical situation, which in turn impacted their formulation and combination of equations. Therefore, this may explain why for the sequential synthesis tasks, we did not observe prominent effects of mathematical complexity on the students' formulation and combination of equations.

In contrast, the simultaneous synthesis problems include concurrent events such that it is necessary to consider all of them at one time. The concurrence of the events may have made it difficult for students to fully unpack the situation thus negatively influencing their formulation and combination of equations. As evidenced by the individual interviews, for the simple version of the task, the students recognized part of the multiple events and part of the multiple pertinent concepts. For the complex version, they oversimplified a multiple events situation to a single event one and used one concept. The individual interviews also indicate that for the simple task, the students formulated equations for part of the pertinent concepts. Although they combined their individual equations, they identified part of the necessary connecting variables for problem solving. For the complex task, they formulated one equation for the one pertinent concept identified. Thus, they did not have to combine multiple equations to solve the problem. In this case, both the characteristics of the simultaneous synthesis tasks and mathematical complexity seemed to impede the students' mathematical performance. Mathematical complexity may have worsened the situation and negatively interfered with the students' formulation and combination of equations. Cognitive load [50] may also account for why students were better able to formulate equations for the sequential synthesis tasks compared to the simultaneous problems. For the sequential tasks, the fact that students could consider one event at a time might have resulted in reduced cognitive load and supported their identification of all the pertinent concepts for formulating the equations. For the simultaneous tasks, the students had to consider the events concurrently which may have negatively interfered with their ability to consider and process all the pertinent information in their working memory. This additional cognitive load may have resulted in a reduced ability to identify the pertinent principles for formulating the equations.
Further, the different results we observed in the sequential and simultaneous tasks may relate to the fact that the variation in the mathematical complexity within each synthesis type was different. For each version of the sequential synthesis task, the students had to handle one equation with one unknown plus three equations with two or three unknowns in each (see Table I). This means that the variation in the mathematical complexity across the three sequential synthesis tasks was not significantly large. For the simple simultaneous task, students could find, at the onset, a value from the linear and angular momentum conservation equation respectively. They were then left to tackle only the energy conservation equation having one unknown. For the complex version, they needed to concurrently manipulate three equations with two or three unknowns in each equation (see Table II). The difference in mathematical complexity difficulty was much larger between the simple and complex simultaneous tasks compared to the difference among the three versions of the sequential problem. This difference in mathematical complexity difficulty may explain the differences observed across the three levels (formulation, combination and simplification of equations) in the simultaneous tasks but not in the sequential problems.

We also made a comparison between the two types of synthesis problems. The findings show that regardless of the task's mathematical complexity, the students were more successful at formulating all the pertinent equations for the sequential synthesis problems compared to the simultaneous tasks. However, once they formulated the fundamental equations, the students were better able to combine the equations and simplify the combined equations for the simultaneous synthesis tasks than for the sequential problems.

There are two possible reasons to explain why students were more successful at formulating the pertinent equations for the sequential problems compared to the simultaneous tasks. The first one pertains to the students' familiarity with and confidence in the concepts underlying the two types of synthesis problems. The second reason deals with the characteristics of the sequential and simultaneous synthesis tasks (i.e., chronological versus concurrent occurrence of events) which may support or impede the students' identification of pertinent concepts. If students fail to identify the pertinent principles, they will not be able to formulate the fundamental equations. These two possible reasons are highlighted in detail in our separate paper investigating the effect of mathematical complexity on students' conceptual performance in the two synthesis problem types [45].

The differences in students' ability to combine and simplify their equations between the two types of synthesis problems can be explained by the differences in the mathematical composition of the equation systems. For the sequential synthesis tasks, the students tackled four fundamental equations involving trigonometry. They were 
required to use trigonometric identity to solve the complex version of the problem. Moreover, they had to combine the equations twice. One is merging the projectile motion equations to find or eliminate time. The other is combining the energy conservation with the projectile motion equations. Our results indicate that after combining the projectile motion equations, mainly for the intermediate and complex tasks, a complicated quadratic equation with trigonometry was obtained. The complexity of the quadratic equations increased when the equations were left in symbolic forms. Further, the students failed to apply their mathematical knowledge of trigonometric identity.

In contrast, for the simultaneous synthesis tasks, the students manipulated three fundamental equations. These equations did not involve trigonometry. However, the students had to use factorization and expansion of algebraic expressions to solve the complex task. Combination of equations occurred only once by plugging the linear and angular momentum conservation equations in the energy conservation expression. Our findings show that the students properly dealt with the linear and quadratic expressions, and they successfully applied their mathematical knowledge of factorization and expansion of algebraic expressions.

In short, the equations for the sequential tasks were more complicated than for the simultaneous problems. However, our results show that although the students failed to mathematically combine and simplify the equations for the sequential tasks, they correctly explained, at a conceptual level, how to obtain the intermediate and final variables from the fundamental equations.

These outcomes add to the literature on students' handling of mathematics during physics problem solving. Previous studies in this research area [3,15-18] have reported that students, in either lower or upper division physics, have difficulties applying sophisticated mathematical tools such as integrals and partial derivatives in advanced physics topics. Nearly all of these studies utilized typical textbooklike problems. Further, there is a dearth of studies investigating university students' handling of algebra problems when solving physics problems [14,24]. In our study, we used synthesis problems, based on fundamentals of energy conservation, projectile motion, linear, and angular momentum conservation which are extensively covered both at the introductory and sophomore level. In addition, these synthesis problems only required basic mathematical skills such as factorization and expansion of algebraic expressions and use of trigonometric identity. Overall, regardless of the level of sophistication of the topic and mathematical tools, the students struggled to apply their mathematical knowledge in the physics context.

Moreover, in the physics domain, "problem-solving expertise should include opportunistically blending of conceptual and formal mathematical reasoning even while manipulating equations." [4]. However, the many instructional materials or strategies designed to improve the problem-solving process have mainly emphasized conceptual reasoning when formulating equations and evaluating the final answer $[4,6,36-39,41]$. Further, these instructional materials have predominantly utilized single-concept problems. We advocate that synthesis problems may provide an additional opportunity to incorporate conceptual reasoning, specifically while manipulating the mathematics. Once the multiple equations are formulated, the students are required to combine them to solve for an answer. As such, they need to have a good sense of the connection between the multiple events, concepts as well as the equations. In short, synthesis problems may emphasize expertlike problem solving approaches among students by channeling their attention to conceptual reasoning when dealing with the conceptual and mathematical aspects of the problem.

\section{ACKNOWLEDGMENTS}

This work is supported by the National Science Foundation (Grant No. DRL-1252399).

\section{APPENDIX A: TYPICAL SOLUTIONS FOR SEQUENTIAL SYNTHESIS PROBLEMS}

For the three sequential problems, we need the four equations:

Energy conservation : $\frac{1}{2} k x^{2}=\frac{1}{2} m v^{2}+m g(L+x) \sin \theta$

Projectile motion : $R=v \cos \theta t$

$$
\begin{aligned}
& y=v \sin \theta t-\frac{1}{2} \mathrm{~g} t^{2} \\
& \tan \phi=\frac{-y}{R}
\end{aligned}
$$




Simple task
From Eq. (A1):
$\quad \frac{m v^{2}}{2}=\frac{k x^{2}}{2}-m g(L+x) \sin \theta$
$\quad v=\left[\frac{k x^{2}}{m}-2 g(L+x) \sin \theta\right]^{\frac{1}{2}}$

Plug values give $v=2.87 \mathrm{~m} / \mathrm{s}$.

Plug Eqs. (A2) and (A4) in Eq. (A3):

$$
-v \cos \theta t \tan \phi=v \sin \theta t-\frac{g t^{2}}{2}
$$

Simplify and isolate for $t$ $t=\frac{2 v(\cos \theta \tan \phi+\sin \theta)}{g}$

Plug values gives $t=0.560 \mathrm{~s}$

From Eq. (A2), plug values gives $R=(2.87) \cos 35(0.560)=1.3 \mathrm{~m}$.
Intermediate task

From Eq. (A2): $t=\frac{R}{v \cos \theta}$

Plug Eq. (A4) and $t=\frac{R}{v \cos \theta}$ in Eq. (A3):

$-R \tan \phi=v \sin \theta\left(\frac{R}{v \cos \theta}\right)-\frac{\mathrm{g} R^{2}}{2 v^{2} \cos ^{2} \theta}$

Simplify and isolate for $v$

$$
v=\left[\frac{g R}{2 \cos ^{2} \theta(\tan \phi+\tan \theta)}\right]^{\frac{1}{2}}
$$

Plug values gives $v=2.87 \mathrm{~m} / \mathrm{s}$

From Eq. (A1): plug $v=2.87 \mathrm{~m} / \mathrm{s}$ and other values and simplify: $1500 x^{2}-11.24 x-13.74=0$

Isolate for $x$

$$
x=\frac{11.24 \pm \sqrt{(11.24)^{2}-4(1500)(-13.74)}}{2(1500)}
$$$$
x=0.1 \mathrm{~m} \text {. }
$$

Complex task

From Eq. (A1):

$v^{2}=\frac{k x^{2}}{m}-2 g(L+x) \sin \theta$

Plug values gives

$v^{2}=15-11.76 \sin \theta \mathrm{m} / \mathrm{s}$.

From Eq. (A2): $t=\frac{R}{v \cos \theta}$

Plug Eq. (A4) and $t=\frac{R}{v \cos \theta}$ in Eq. (A3) and simplify:

$$
\tan \phi=\tan \theta-\frac{g R}{2 v^{2} \cos ^{2} \theta}
$$

Plug values and expression for $v^{2}$ and simplify:

\section{APPENDIX B: TYPICAL SOLUTIONS FOR SIMULTANEOUS SYNTHESIS PROBLEMS}

For both versions of the simultaneous problem, we need the three equations:

Energy conservation : $\frac{1}{2} k \Delta x^{2}+\frac{1}{2}(M+m) v_{o}^{2}=\frac{1}{2} m v_{p}^{2}+\frac{1}{2} M v_{f}^{2}+\frac{1}{2} I \omega^{2}$

Linear momentum conservation: $(M+m) v_{o}=M v_{f}+m v_{p}$

Angular momentum conservation : $m v_{o} R=m v_{p} R+I \omega$

Simple task

From Eq. (B2):

$v_{f}=\frac{(M+m) v_{o}-m v_{p}}{M}$

Plug values gives $v_{f}=-7 \mathrm{~m} / \mathrm{s}$

From Eq. (B3):

$\omega=\frac{m R v_{o}-m R v_{p}}{\frac{1}{2} m R^{2}}$

Plug values gives $\omega=-32 \mathrm{rad} / \mathrm{s}$

From Eq. (B1), simplify and isolate for $\Delta x$ :

$\Delta x= \pm \sqrt{\frac{m v_{p}^{2}+M v_{f}^{2}+\frac{1}{2} M R^{2} \omega^{2}-(M+m) v_{o}^{2}}{k}}$

Plug values gives

$$
\Delta x= \pm 0.176 \mathrm{~m}, \Delta x=17.6 \mathrm{~cm}
$$

Intermediate task

From Eq. (B2):

$v_{f}=\frac{(M+m) v_{o}-m v_{p}}{M}$

From Eq. (B3):

$$
\omega=\frac{m v_{o} R-m v_{p} R}{I}=\frac{m R}{I}\left(v_{o}-v_{p}\right)
$$

Plug expressions for $v_{f}, \omega$, and $I=1 / 2 M R^{2}$ in Eq. (B1):

$$
\frac{1}{2} k \Delta x^{2}+\frac{1}{2}(M+m) v_{o}^{2}=\frac{1}{2} m v_{p}^{2}+\frac{1}{2} \frac{M}{m^{2}}\left[(M+m) v_{o}-m v_{p}\right]^{2}+\frac{1}{2} I\left(\frac{m R}{I}\right)^{2}\left(v_{o}-v_{p}\right)^{2}
$$

Plug values, simplify, and isolate for $v_{o}$ :

$$
\begin{gathered}
(-42) v_{o}^{2}+(924) v_{o}-3581.375=0 \\
v_{o}=\frac{22 \pm \sqrt{(22)^{2}-4(85.27)}}{2} \\
v_{o}=(5.0 \mathrm{~m} / \mathrm{s}, 17 \mathrm{~m} / \mathrm{s}), v_{o}=5.0 \mathrm{~m} / \mathrm{s} .
\end{gathered}
$$




\section{APPENDIX C: CODING SCHEME FOR} SEQUENTIAL SYNTHESIS PROBLEMS

Level I: Formulation of equations - deals with identifying all the pertinent concepts and generating equations to mathematically express each pertinent concept, energy conservation and projectile motion.

If a student formulates all the equations for all the pertinent concepts, then the student is considered to be in this category. We coded for the following:

- correct energy conservation equation linking the relevant types of energy for the initial and final conditions and using the correct height expression for the gravitational potential energy term, i.e., $1 / 2 k x^{2}=1 / 2 m v^{2}+m g(L+x) \sin \theta$.

- incorrect energy conservation equations linking irrelevant types of energy for the initial and final conditions and/or using incorrect height expressions for the gravitational potential energy term.

- correct projectile motion equations: (i) horizontal motion, $R=v \cos \theta t$, (ii) trigonometry relating angle $\phi$, magnitude and direction of the horizontal and vertical motion, $\tan \phi=-y / R$, and (iii) equation for vertical motion, $-y=-R \tan \phi=v \sin \theta t-1 / 2 g t^{2}$.

- incorrect projectile motion equations such as incorrect (i) initial and/or final position in the vertical motion equation, (ii) expressions for the components of the block's velocity, and (iii) expressions for trigonometry.

Level II: Combination of mathematical equationsrefers to combining the equations emerging on application of all the pertinent concepts.

Students who did combine their formulated energy conservation and projectile motion equations are in this category. The students tend to merge the expression for the block's velocity from the energy conservation equation with the vertical motion equation.

Level III: Simplification of equation to obtain the variable of interest-pertains to students' attempt to identify variables and rearrange equations such that the required variable is written as a function of the others.

Students who did attempt to simplify their expression, emerging from combining the energy conservation and projectile motion equations, to determine the required variable are in this category. From the expression emerging from combining the energy conservation and projectile motion equations, the required variable is (i) $R$, the horizontal distance, for the simple task, (ii) $x$, the initial compression of the spring, for the intermediate version, and (iii) $\theta$, the angle of initial slope, for the complex task.

\section{APPENDIX D: CODING SCHEME FOR SIMULTANEOUS SYNTHESIS PROBLEMS}

Level I: Formulation of equations: deals with identifying all the pertinent concepts and generating equations to mathematically express each pertinent concept, energy conservation, linear, and angular momentum conservation.

If a student formulates all the equations for all the pertinent concepts, then the student is considered to be in this category. We coded for the following:

- correct linear momentum conservation equation linking the initial and final linear momentum due to the disc and the projectile. We also considered the correct velocity variables used for the initial and final conditions i.e., $(M+m) v_{o}=M v_{f}+m v_{p}$.

- correct angular momentum conservation equation linking the initial and final angular momentum due to the projectile, and the final angular momentum due to the disc. We also considered the correct velocity variables used for the initial and final conditions, i.e., $m v_{o} R=m v_{p} R+I \omega$.

- correct energy conservation equation linking the relevant types of energy for the initial and final conditions. We also considered the correct velocity variables used for the initial and final conditions, i.e., $1 / 2 k \Delta x^{2}+1 / 2(M+m) v_{o}^{2}=1 / 2 M v_{f}^{2}+1 / 2 m v_{p}^{2}+1 / 2 I \omega^{2}$.

- the incorrect equations for energy conservation, linear, and angular momentum conservation relating irrelevant types of energy, linear, and angular momentum, respectively, and/or the incorrect velocity variables used for the initial and final conditions.

Level II: Combination of mathematical equationsrefers to combining the equations emerging on application of all the pertinent concepts.

Students who did combine their formulated equation for energy conservation, linear, and angular momentum conservation are in this category. The students tend to merge the expression for the disc's final velocity $v_{f}$ and angular velocity $\omega$ from the linear and angular momentum conservation equation, respectively, with the energy conservation equation.

Level III: Simplification of equation to obtain the variable of interest-pertains to students' attempt to identify variables and rearrange equations such that the required variable is written as a function of the others.

Students who did attempt to simplify their expression, emerging from combining their energy conservation, linear, and angular momentum conservation equations, to determine the required variable are in this category. From the expression emerging from combining the energy conservation, linear, and angular momentum conservation equations, the required variable is (i) $\Delta x$, the initial compression of the spring, for the simple task, and (ii) $v_{o}$, the disc's initial velocity, for the complex task.

\section{APPENDIX E: INTERVIEW PROTOCOL FOR SEQUENTIAL AND SIMULTANEOUS SYNTHESIS PROBLEMS}

- Tell me, in your own words, what the task is about? 
- Guide me over your solution. How did you proceed to solve this problem?

- When you were given this task, how did you think about it before you started solving it?

- You mentioned that you used the best approach to tackle this problem. Can you tell me more about that?

- You said that you use two different methods; you mentioned that you attempted the problem in stages. Can you explain what you mean by that? Can you elaborate on your response?

- You said you use these concepts. How did you use them to solve the problem?

- I see that you got stuck with the math here. What happened at that point?

- You mentioned mathematically you were having some troubles. Can you tell me more about it?
- Did you find it easy or hard to manipulate the mathematics? Can you elaborate on your response?

- Any difficulties that you face while trying to merge these equations?

- How did you find the task? In what ways did you find it easy or difficult?

\section{APPENDIX F: RESULTS FOR WHY STUDENTS DID NOT COMBINE THE EQUATIONS FORMULATED FOR THE SEQUENTIAL SYNTHESIS TASKS}

Table III presents the actions of those students who did not combine the pertinent equations after formulating them for the sequential synthesis tasks. Illustrations of students' actions for the simple, intermediate and complex sequential synthesis tasks are presented in Fig. 11.

TABLE III. Actions of those students who did not combine the equations across the three versions of the sequential synthesis task.

\begin{tabular}{|c|c|c|c|}
\hline Students' actions & Simple $(N=4)$ & Intermediate $(N=5)$ & Complex $(N=6)$ \\
\hline $\begin{array}{l}\text { 1. The students formulated all the fundamental equations. } \\
\text { However, instead of mathematically manipulating the } \\
\text { equations, they wrote down the key intermediate } \\
\text { variables and steps needed to find the final variable of } \\
\text { interest. }\end{array}$ & 3 & 0 & 2 \\
\hline $\begin{array}{l}\text { 2. The student first tried to solve the problem using } \\
\text { Newton's Second Law before shifting to using energy } \\
\text { conservation and projectile motion. }\end{array}$ & 1 & 0 & 0 \\
\hline $\begin{array}{l}\text { 3. The students merged the projectile motion equations. } \\
\text { They obtained a complicated quadratic equation with } \\
\text { trigonometry for the intermediate and complex tasks. } \\
\text { These expressions were left in purely symbolic forms. } \\
\text { The students did not combine the expressions although } \\
\text { they knew they needed to do so to find the variable of } \\
\text { interest. This is indicated by the students' drawing an } \\
\text { arrow linking the expressions from the energy } \\
\text { conservation and projectile motion equations, and re- } \\
\text { arranging the energy conservation equation with } \\
\text { velocity as the required variable. The students also re- } \\
\text { arranged the projectile motion equations with velocity } \\
\text { or time as the necessary variable. }\end{array}$ & 0 & 5 & 4 \\
\hline
\end{tabular}




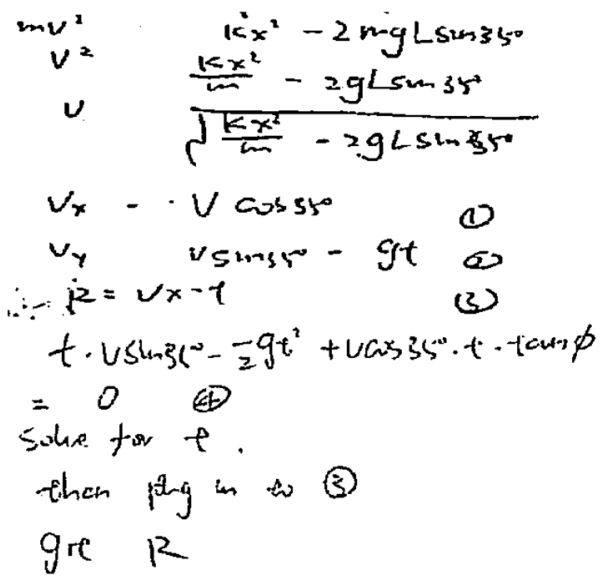

(a)

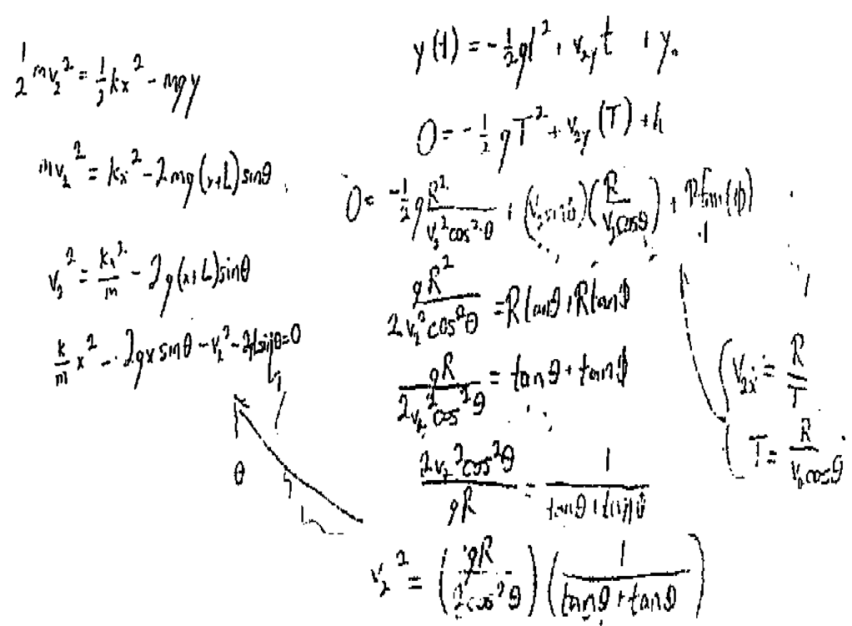

(b)

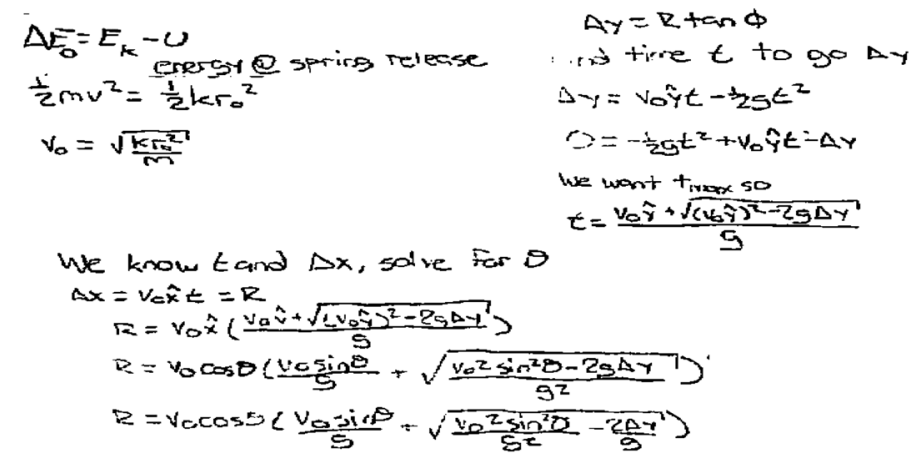

(c)

FIG. 11. Written solutions of those students who failed to combine their formulated equations for the (a) simple, (b) intermediate, and (c) complex sequential synthesis tasks.

\section{APPENDIX G: RESULTS FOR WHY STUDENTS DID NOT SIMPLIFY THE COMBINED EQUATIONS FOR THE SEQUENTIAL SYNTHESIS TASKS}

Table IV highlights the actions of those students who did not simplify the equations after combining them for the sequential synthesis tasks. Figure 12 depicts examples of students' actions for the simple, intermediate and complex sequential synthesis tasks.

TABLE IV. Actions of those students who did not simplify their equations after combining them for the simple, intermediate and complex sequential synthesis tasks.

\begin{tabular}{|c|c|c|c|}
\hline Students' actions & Simple $(N=2)$ & Intermediate $(N=5)$ & Complex $(N=11)$ \\
\hline $\begin{array}{l}\text { 1. From the energy conservation equation, the students found } \\
\text { an expression rather than a value for velocity. They } \\
\text { combined the } v \text { expression with the projectile motion } \\
\text { equations to obtain a quadratic expression involving } \\
\text { trigonometry. Although all the variables were known, the } \\
\text { students did not plug in the values to simplify the equation } \\
\text { to solve for the variable of interest. }\end{array}$ & 2 & 0 & 0 \\
\hline
\end{tabular}


TABLE IV. (Continued)

\begin{tabular}{l}
\hline \hline Students' actions \\
$\begin{array}{l}\text { 2. The students obtained an expression for velocity from the } \\
\text { energy conservation equation. They combined this }\end{array}$ \\
expression with the horizontal and the vertical motion \\
equation respectively. Two intricate quadratic expressions \\
with trigonometry resulted for the intermediate task. These \\
expressions were left in symbolic form. They had to be \\
tackled simultaneously to find the variable of interest. \\
3. The students obtained an intricate quadratic expression for \\
the complex task after combining the energy conservation \\
and projectile motion equations. They left the equations in \\
symbolic form and had to use trigonometric identity, \\
which they failed to apply, to find the variable of interest. \\
They may also write down that they were unsure how to \\
solve for the variable of interest from the combined \\
equation.
\end{tabular}

$$
\begin{aligned}
& \frac{1}{2} k x^{2}=\frac{1}{2} m v^{2}+m g l \sin \theta \\
& V=\sqrt{\frac{2\left(\frac{1}{2} k \lambda^{2}-m g l \sin \theta\right)}{m}} \quad V_{y}=V_{\cos \theta} \quad V_{y}=V_{\sin } \theta \\
& \begin{array}{l}
-R \sin \phi=0+v_{y} t-\frac{1}{2} g t^{2} \\
-\frac{1}{2} g t^{2}+V_{y} t+R \sin \phi
\end{array} \\
& t=\frac{-V_{y} \pm \sqrt{V^{2}-4\left(-\frac{1}{5} g\right)(p \sin \phi)}}{2\left(\frac{1}{2}\right)} \\
& \text { Now plug into } x=V t \text { whers } x-R \quad \xi V=V_{x}
\end{aligned}
$$

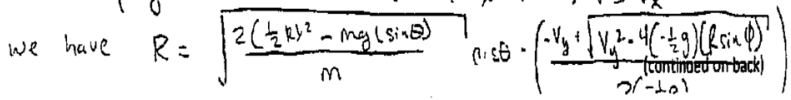

(a)

$$
\begin{aligned}
& K E=E_{s}-\Delta V_{1}=\frac{1}{2} k x^{2}-m g h=\frac{1}{2} m V^{2} \\
& v=\sqrt{\frac{\frac{1}{2} \Delta x^{2}-(L+x) \operatorname{sen} \theta m}{\frac{1}{2} m}}=\sqrt{\frac{x}{x_{1} x^{2}-2(L+x) \sin \theta g}} \\
& y(t)-v_{y} t+y_{0}=\cos \theta \cdot \sqrt{\frac{x_{n}}{x^{2}-2 a+x / \sin 0 g}}+ \\
& z(f)=2+v_{z}(t)+-\frac{1}{2} g t^{2}=\sin \theta \sqrt{\frac{A}{m} x^{2}-2(2+x) \sin \theta_{y}}+-\frac{1}{2} y t^{2} \\
& \text { At tere to we want } \quad x\left(t_{c}\right)=R \text { and } z\left(t_{0}\right)=-R \tan \phi \\
& F\left(t_{0}\right)=R \text { and } E\left(t_{0}\right)=-R \tan \phi \\
& \text { Since fo is ches the wes bils the slope again }
\end{aligned}
$$

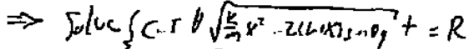

$$
\begin{aligned}
& \left\langle\sin \theta \sqrt{\frac{k}{m}} \overline{x^{2}-2 \cos \times \operatorname{ls} s \theta g}+-\frac{1}{2}, t^{2}=-R \tan \phi\right.
\end{aligned}
$$

(b)

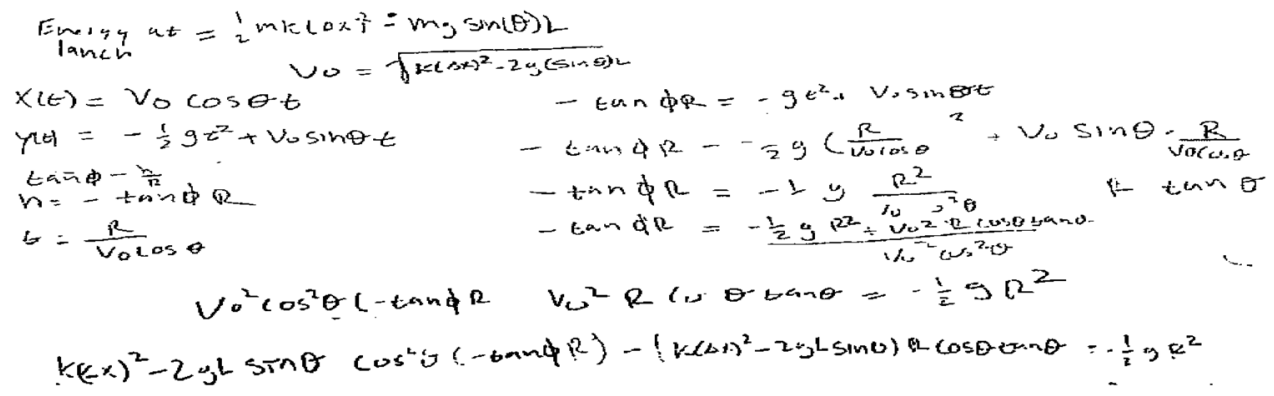

(c)

FIG. 12. Written solutions of those students who failed to simplify their combined equations for the (a) simple, (b) intermediate, and (c) complex sequential synthesis tasks. 


\section{APPENDIX H: RESULTS FOR WHY STUDENTS COMBINED AND SIMPLIFIED THE EQUATIONS FOR THE SIMULTANEOUS SYNTHESIS TASKS}

The actions of those students who were able to combine and simplify the equations for the simple and the complex simultaneous synthesis task are presented in Table V. Examples of students' actions for the simple and complex simultaneous synthesis tasks are presented in Fig. 13.

TABLE V. Actions of those students who combined and simplified the equations formulated for the simple and complex simultaneous synthesis tasks.

\begin{tabular}{lc}
\hline \hline Students' actions & Simple $(N=11)$ \\
\hline 1. The students found a value for the disc's final linear velocity and & 11 \\
angular velocity from the linear momentum conservation and & 0 \\
angular momentum conservation equation respectively. They then & \\
plugged these two values in the energy conservation equation. This \\
resulted in a quadratic expression which was straightforward to \\
manipulate to find a value for the variable of interest. \\
2. The students found an expression for the disc's final linear velocity \\
and angular velocity from the linear momentum conservation and \\
angular momentum conservation equation respectively. These two \\
expressions were linear. They then substituted these two expressions \\
in the energy conservation equation. This resulted in a quadratic \\
expression requiring the use of expansion and factorization of \\
algebraic expressions, which they successfully applied, to find the \\
variable of interest.
\end{tabular}

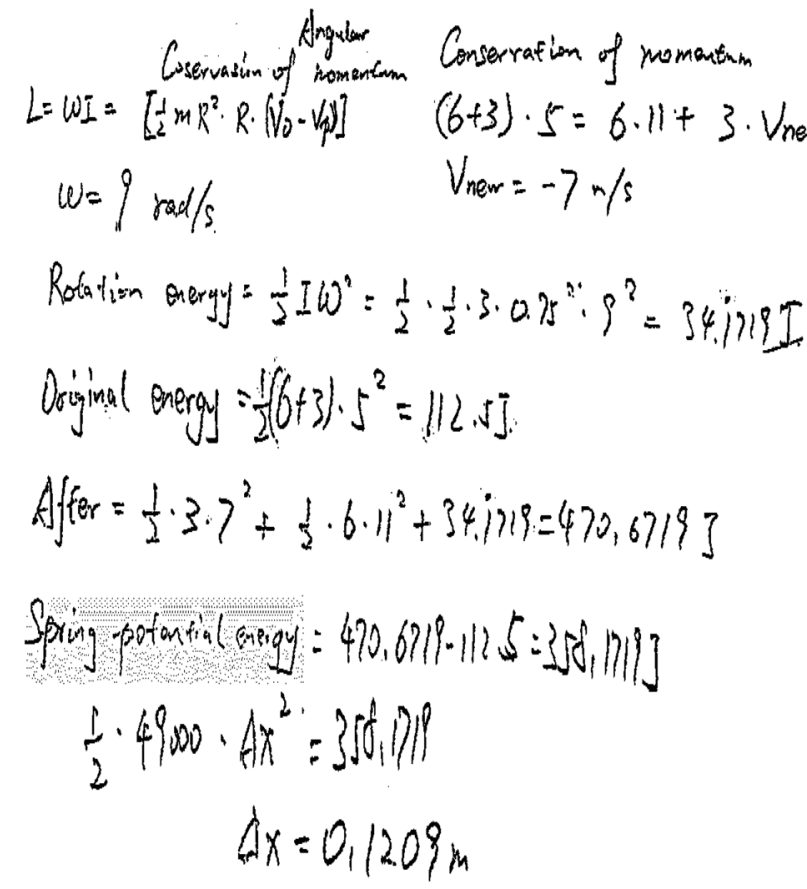

(a)

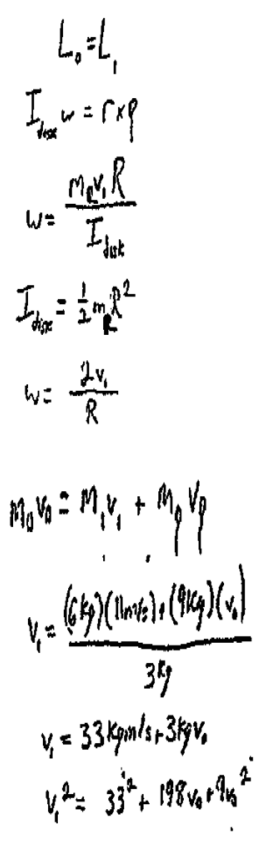

$$
\begin{aligned}
& U_{s 0}+K_{0}=K_{1}+K_{p}+K_{\text {not }} \\
& \frac{1}{2} k x^{2}+\frac{1}{2} m v_{1}^{2}=\frac{1}{2} m w^{2}+\frac{1}{2} n v_{p}{ }^{2}+\frac{i}{2} I_{\omega} \omega^{2} \\
& k_{x}{ }^{2}+m_{v} v_{2}^{2}=m v_{1}^{2}+m_{p} v_{p}^{2}+\left(\frac{1}{2} m_{x} R^{2}\right)\left(\frac{2 v_{1}}{R}\right)^{2} \\
& m_{1} v_{0}^{2}-2 m_{p} v_{1}^{2}=m_{p} v_{p}^{2}-k_{x}^{2}
\end{aligned}
$$

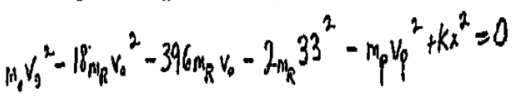$$
(\underbrace{\left(m_{i}-18 m_{k}\right) v_{0}^{2}}+\underbrace{\left(-3966 m_{g}\right) v_{0}}+\underbrace{\left(-2-2 m_{R} 33^{2}-m r_{p} v_{p}^{2}+k x^{2}\right)})=0
$$$$
a=-45 \quad b=-2376 \quad c=1375
$$$$
V_{0}=-53.347 \mathrm{~m} / \mathrm{s}
$$$$
\text { or }
$$$$
V_{0}=.548 \mathrm{~m} / \mathrm{s}
$$

(b)

FIG. 13. Written solutions of those students combining the formulated equations and simplifying the combined equations for the (a) simple, and (b) complex simultaneous synthesis tasks. 
[1] E. F. Redish, Proceedings of the Conference on World View on Physics Education in 2005: Focusing on Change, Delhi, India, 2005, https://arxiv.org/ftp/physics/papers/ 0608/0608268.pdf.

[2] T. J. Bing and E. F. Redish, Analyzing problem solving using math in physics: Epistemological framing via warrants, Phys. Rev. ST Phys. Educ. Res. 5, 020108 (2009).

[3] R. E. Pepper, S. V. Chasteen, S. J. Pollock, and K. K. Perkins, Observations on student difficulties with mathematics in upper division electricity and magnetism, Phys. Rev. ST Phys. Educ. Res. 8, 010111 (2012).

[4] E. Kuo, M. M. Hull, A. Gupta, and A. Elby, How students blend conceptual and formal mathematical reasoning in solving physics problems, Sci. Educ. 97, 32 (2013).

[5] A. Collins and W. Ferguson, Epistemic forms and epistemic games: structures and strategies to guide inquiry, Educ. Psychol. 28, 25 (1993).

[6] J. Tuminaro and E. F. Redish, Elements of a cognitive model of physics problem solving: Epistemic games, Phys. Rev. ST Phys. Educ. Res. 3, 020101 (2007).

[7] K. E. Black and M. C. Wittmann, Epistemic games in integration: Modeling resource choice, AIP Conf. Proc. 951, 53 (2007).

[8] E. C. Sayre and M. C. Wittmann, Plasticity of intermediate mechanics students' coordinate system choice, Phys. Rev. ST Phys. Educ. Res. 4, 020105 (2008).

[9] M. C. Wittmann and K. E. Black, Mathematical actions as procedural resources: An example from the separation of variables, Phys. Rev. ST Phys. Educ. Res. 11, 020114 (2015).

[10] D. Hammer, A. Elby, R. E. Scherr, and E. F. Redish, in Transfer of Learning: From a Modern Multidisciplinary Perspective, edited by J.P. Mestre (Information Age Publishing, Greenwich, CT, 2005).

[11] D. Hu and N.S. Rebello, Shifting college students' epistemological framing using hypothetical debate problems, Phys. Rev. ST Phys. Educ. Res. 10, 010117 (2014).

[12] T. J. Bing and E. Redish, The cognitive blending of mathematics and physics knowledge, AIP Conf. Proc., 883, 26 (2006).

[13] D. Hu and N.S. Rebello, Using conceptual blending to describe how students use mathematical integrals in physics, Phys. Rev. ST Phys. Educ. Res. 9, 020118 (2013).

[14] S. W. Brahmia, A. Boudreaux, and S. E. Kanim, Obstacles to mathematization on introductory physics, arXiv: 1601.01235 .

[15] F. R. Yeats and J. R. Hundhausen, Calculus and physics: Challenges at the interface, Am. J. Phys. 60, 716 (1992).

[16] D. Nguyen and N. S. Rebello, Students' difficulties with integration in electricity, Phys. Rev. ST Phys. Educ. Res. 7, 010113 (2011).

[17] D. Hu and N. S. Rebello, Understanding student use of differentials in physics integration problems, Phys. Rev. ST Phys. Educ. Res. 9, 020108 (2013).

[18] B. R. Wilcox and S. J. Pollock, Upper-division student difficulties with separation of variables, Phys. Rev. ST Phys. Educ. Res. 11, 020131 (2015).

[19] J. R. Thompson, C. A. Manogue, D. J. Roundy, and D. B. Mountcastle, Representations of partial derivatives in Thermodynamics, AIP Conf. Proc. 1413, 85 (2012).
[20] J. R. Thompson, B. R. Bucy, and D. B. Mountcastle, Assessing student understanding of partial derivatives in Thermodynamics, AIP Conf. Proc. 818, 77 (2006).

[21] B. R. Bucy, J. R. Thompson, and D. B. Mountcastle, Student (mis) application of partial differentiation to material properties, AIP Conf. Proc. 883, 157 (2007).

[22] E. B. Pollock, J. R. Thompson, and D. B. Mountcastle, Student understanding of the physics and mathematics of process variables in $P-V$ diagrams, AIP Conf. Proc. 951, 168 (2007).

[23] W. M. Christensen and J. R. Thompson, Investigating student understandings of physics concepts and the underlying calculus concepts in thermodynamics, Proceedings of the 13th Annual Conference on Research in Undergraduate Mathematics Education (Mathematical Association of America, Oberlin, OH, 2010).

[24] B. L. Sherin, How students understand physics equations, Cognit. Instr. 19, 479 (2001).

[25] J. Tuminaro, Ph.D. thesis, University of Maryland, 2004.

[26] O. Uhden, R. Karam, M. Pietrocola, and G. Pospiech, Modelling mathematical reasoning in physics education, Sci. Educ. 21, 485 (2012).

[27] R. Karam, Framing the structural role of mathematics in physics lectures: a case study on electromagnetism, Phys. Rev. ST Phys. Educ. Res. 10, 010119 (2014).

[28] R. Lopez-Gay, J. Martínez Sáez, and J. Martínez Torregrosa, Obstacles to mathematization in physics: the case of the differential, Sci. Educ. 24, 591 (2015).

[29] E. F. Redish and K. A. Smith, Looking beyond content: Skill development for engineers, J. Eng. Educ. 97, 295 (2008).

[30] C. A. Ogilvie, Changes in students' problem-solving strategies in a course that includes context-rich, multifaceted problems, Phys. Rev. ST Phys. Educ. Res. 5, 020102 (2009).

[31] P. D. Antonenko, C. A. Ogilvie, D. S. Niderhauser, J. Jackman, P. Kumsaikaew, R. R. Marathe, and S. M. Ryan, Understanding student pathways in context-rich problems, Educ. Inf. Technol. 16, 323 (2011).

[32] R. Qing Xu, Ph.D. dissertation, University of Minnesota, 2013 (unpublished).

[33] S. Lin and C. Singh, Using isomorphic problems to learn introductory physics, Phys. Rev. ST Phys. Educ. Res. 7, 020104 (2011).

[34] L. Ding, N. Reay, A. Lee, and L. Bao, Exploring the role of conceptual scaffolding in solving synthesis problems, Phys. Rev. ST Phys. Educ. Res. 7, 020109 (2011).

[35] A. Van Heuvelen, Learning to think like a physicist: A review of research-based instructional strategies, Am. J. Phys. 59, 891 (1991).

[36] A. Van Heuvelen, Overview, case study physics, Am. J. Phys. 59, 898 (1991).

[37] A. Van Heuvelen and X. Zou, Multiple representations of work-energy processes, Am. J. Phys. 69, 184 (2001).

[38] W. J. Leonard, R. J. Dufresne, and J. P. Mestre, Using qualitative problem-solving strategies to highlight the role of conceptual knowledge in solving problems, Am. J. Phys. 64, 1495 (1996).

[39] R. J. Dufresne, W. J. Gerace, and W. J. Leonard, Solving physics problems with multiple representations, Phys. Teach. 35, 270 (1997). 
[40] F. Reif, Milikan Lecture 1994: Understanding and teaching important scientific thought processes, Am. J. Phys. 63, 17 (1995).

[41] D. P. Maloney, Getting started in PER, Reviews in PER (American Association of Physics Teachers College Park, MD, 2011), Vol. 2.

[42] D. R. White, R. Badeau, A. F. Heckler, and L. Ding, Bottlenecks in Solving Synthesis Problems, in Proceedings of the Physics Education Research Conference 2014, Minneapolis, MN, edited by P. V. Engelhardt, A. D. Churukian, and D. L. Jones (AIP, New York, 2014), .

[43] L. Ding, N. W. Reay, A. Lee, and L. Bao, Using conceptual scaffolding to foster effective problem solving, AIP Conf. Proc. 1179, 129 (2009).

[44] L. Ding, N. W. Reay, A. F. Heckler, and L. Bao, Sustained Effects of Solving Conceptually-scaffolded Synthesis Problems, AIP Conf. Proc. 1289, 133 (2010).

[45] B. Ibrahim, L. Ding, A. F. Heckler, D. R. White, and R. Badeau, Student's conceptual performance on synthesis physics problems with varying mathematical complexity, Phys. Rev. Phys. Educ. Res. 13, 010133 (2017).
[46] K. R. Koedinger, M. W. Alibali, and M. J. Nathan, Trade-offs between grounded and abstract representations: evidence from algebra problem solving, Cogn. Sci. 32, 366 (2008).

[47] K. R. Koedinger and B. A. MacLaren, Developing a pedagogical domain theory of early algebra problem solving (2002), Citeseerx.ist.psu.edu/viewdoc/summary? doi=10.1.1.139.8036.

[48] N. Heffernan and K. R. Koedinger, The composition effect in symbolizing: The role of symbol production vs text comprehension, in Proceedings of the Nineteenth Annual Conference of the Cognitive Science Society, edited by M. G. Shafto and P. Langley (Lawrence Erlbaum Associates, Hillsdale, NJ, 1997), pp. 307-312.

[49] A. F. Heckler and E. C. Sayre, What happens between preand post-tests: Multiple measurements of student understanding during an introductory physics course, Am. J. Phys. 78, 768 (2010).

[50] J. Sweller, Cognitive load during problem solving: Effects on learning, Cogn. Sci. 12, 257 (1988). 